\title{
Comparative morphology of the endophallic structures of the genus Laius (Coleoptera, Melyridae), with the descriptions of three new species
}

\author{
Hiroyuki YOSHITOMI \\ Ehime University Museum, Bunkyo 3, Matsuyama, Ehime Pref. 790-8577, Japan. \\ E-mail: hymushi@agr.ehime-u.ac.jp (corresponding author) \\ urn:1sid:zoobank.org:author:744FA6FC-2D84-41B7-8A6C-C1611CC68EE1
}

\begin{abstract}
The endophallic structure of the genus Laius is studied and discussed based on the examination of 19 species from Asia to the Indian Ocean. The structure contains two primary sclerites (named gonoporal piece and ligula), a secondary sclerite on the basal part of the gonoporal piece (named additional sclerite) in some species, and a membranous basal area closely covered with many spines (named spinous area). Five species groups are recognized based on the morphology of the endophallic sclerites. The sympatric species have different body sizes and quite distinguishable endophallic sclerites (= different species group), while the allopatric species have overlapping body sizes and similar endophallic sclerites (= same species group). Three new species are described and six previously known species are redescribed with endophallic sclerites, and the descriptions of endophallic sclerites of the remaining ten species are added. The larva of Laius rodriguesensis sp. nov. is also described. The genus Nossibeus Evers, 1994 is synonymised with Laius Guérin-Méneville, 1830.
\end{abstract}

Keywords. Melyridae, taxonomy, distribution, endophallus, new species.

Yoshitomi H. 2014. Comparative morphology of the endophallic structures of the genus Laius (Coleoptera, Melyridae), with the descriptions of three new species. European Journal of Taxonomy 97: 1-29. http://dx.doi. org/10.5852/ejt.2014.97

\section{Introduction}

Male genitalia are considered one of the most important diagnostic traits in insect systematics (Simmons 2014), and this also applies to the order Coleoptera (e.g., Sharp \& Muir 1912). Some groups in this order have developed endophallic structures (e.g., sclerites, projections, small denticles), and these characters provide important information both taxonomically and phylogenetically. The endophallic structures are mainly observed in relatively large body-sized beetle families (e.g., Carabidae, Cerambycidae, Chrysomelidae), because it is necessary to evert and inflate the endophallus to observe the structures (Sasakawa 2005; Yamasako \& Ohbayashi 2011). Therefore, it is difficult to dissect and observe the endophallic structures in smaller-sized groups.

The melyrid genus Laius Guérin-Méneville, 1830 is represented by about 30 species from the Pacific Islands, Indian Ocean, Australia, and the eastern coast of Africa (see Appendix); however, numerous species have been erroneously included in this genus and will be transferred to other genera (Plonski 
2013; Wittmer 1997). The genus has a well-developed endophallic structure consisting of many small spines and some large sclerites, and these characters have been observed through the aedeagus (Wittmer 1997; Yoshitomi 2008; also see Fig. 2B). However, the characters could not be well understood or described.

The main aim of this study is to inspect the validity of endophallic structures for the recognition of species in this genus. I firstly define the terminology of the endophallic structures of the genus Laius and secondly describe the endophallic sclerites of 19 species, including the descriptions of three new species and redescriptions of six poorly known species.

\section{Materials and methods}

General observations and dissections were made under a Leica MZ95 stereomicroscope. Microstructures of dissected parts were studied in pure glycerine under an Olympus BH-2 compound microscope. After observation, the dissected parts were mounted on the same card with the specimen. Photographs were taken under a Leica MZ95 using a microscopy camera system (Nikon DS-Fi1-L2), and combined with the automontage software Combine ZM (Alan Hadley, UK). Some structures were observed with an SEM (Hitachi S-225) after coating with gold.

The endophallus was extracted from the ventral part of the aedeagal apex using a pin and tweezers. If the endophallus could not be extracted from the aedeagal apex, endophallic sclerites were directly removed from the endophallus. After observation, the structures were preserved in pure glycerine.

The holotypes and paratypes are preserved in the Ehime University Museum, Matsuyama (EUMJ), Naturhistorisches Museum Wien (NMW), Natural History Museum Basel (NHMB) and Bishop Museum, Hawaii (BPBM).

\section{Abbreviations}

Morphological abbreviations used for measurements are as follows (also see Fig. 2):

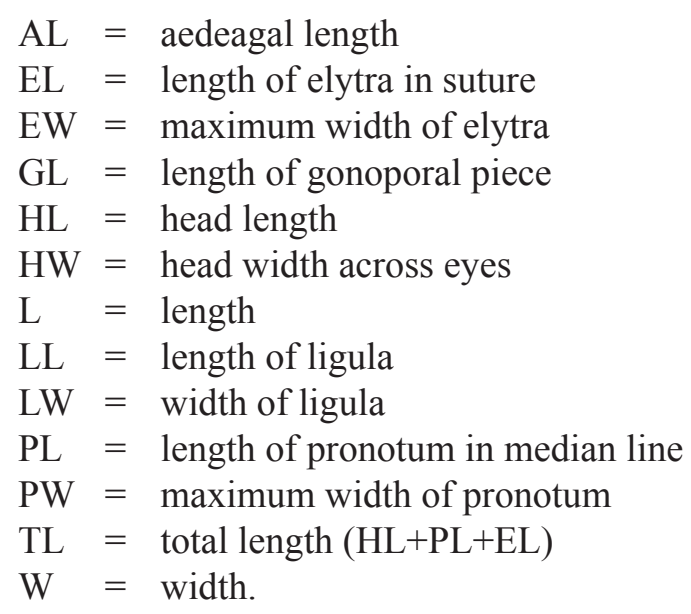

The average is given in parentheses after the range.

\section{Results}

\section{Characteristics and terminology of endophallic structures}

In the copulation position, only the endophallus of the male genitalia is engaged with the female structures (Fig. 1). Endophallic structures (Figs 1-2) consist of a membranous basal area (named spinous area), 
two primary sclerites (named gonoporal piece and ligula), and a secondary sclerite in some species (named additional sclerite).

Spinous area (Figs 2A, 12D): membranous basal area, closely covered with many short spines (Fig. 12D), about $0.5 \times$ length of AL. The differences in this character between species cannot be observed, and the comparison and description of each species is not given in this study.

Gonoporal piece (Fig. 2C; $g p$ in Figs 3-4): longest sclerite, about $0.6 \times$ length of AL, straight to rather curved, with pointed or rounded apex, closely covered with minute spines in basal portion of some species, projecting apically and straight from the apical portion of spinous area.

Ligula (Fig. 2D; $l$ in Figs 3-4): straight to C-shaped curved sclerite, with pointed apex, shorter than gonoporal piece, projecting laterally from the apico-lateral portion of spinous area.

Additional sclerite (as in Figs 3-4): secondary sclerite situated in basal part of gonoporal piece, shorter than ligula, projecting laterally from the basal portion of gonoporal piece.

\section{Taxonomy}

Class Hexapoda Blainville, 1816

Order Coleoptera Linnaeus, 1758

Superfamily Cleroidea Latreille, 1802

Family Melyridae Leach, 1815

Subfamily Malachiinae Fleming, 1821

Genus Laius Guérin-Méneville, 1830

Laius Guérin-Méneville, 1830: 78 [Type species: Laius cyaneus Guérin-Méneville, 1830]. Megadeuterus Westwood, 1833: 678 [Type species: Paussus flavicornis Fabricius, 1801]. Rotamalachius Kôno, 1937 in Kôno \& Esaki 1937: 121 [Type species: Rotamalachius esakii Kôno, 1937]. Nossibeus Evers, 1994: 173 [Type species: Laius politus Fairmaire, 1880]. syn. nov.

\section{Remarks}

The genus Nossibeus was erected for Laius politus Fairmaire, 1880 (Evers 1994). After observation of this species, including its endophallic structure, it was clear that this species cannot be recognised as another species of the genus Laius. In the present paper, Nossibeus Evers, 1994 is treated as a junior synonym of Laius Guérin-Méneville, 1830 (syn. nov.).

Laius baliensis sp. nov. urn:1sid:zoobank.org:act:9D51A886-E14B-4DE6-96C3-410AF3CBC669

Figs $1,3 \mathrm{~A}, 5 \mathrm{~A}-\mathrm{B}, 7 \mathrm{~A}, 8 \mathrm{~A}, 9 \mathrm{~A}, 13 \mathrm{~A}-\mathrm{B}$

Laius sp. - Yoshitomi 2008: 289.

\section{Diagnosis}

Body small, $\mathrm{TL}=4.10-4.46 \mathrm{~mm}$ in male, $4.35-4.75 \mathrm{~mm}$ in female; male antennomere III oval, deeply concave in dorso-mesal portion, $\mathrm{L} / \mathrm{W}=0.95$; aedeagal apex rounded; gonoporal piece straight and slender; ligula slightly curved; additional sclerite absent.

\section{Etymology}

After the type locality, Bali Island, Indonesia. 


\section{Type material}

\section{Holotype}

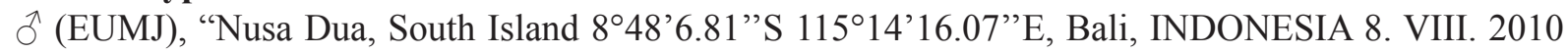
H. Yoshitomi leg.".

\section{Paratypes}

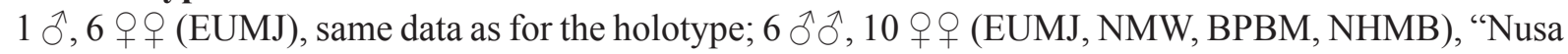
Dua, North Island $8^{\circ} 47^{\prime} 52.72$ "S 105¹4'9.31”'E, Bali, INDONESIA 7-8. VIII. 2010 H. Yoshitomi

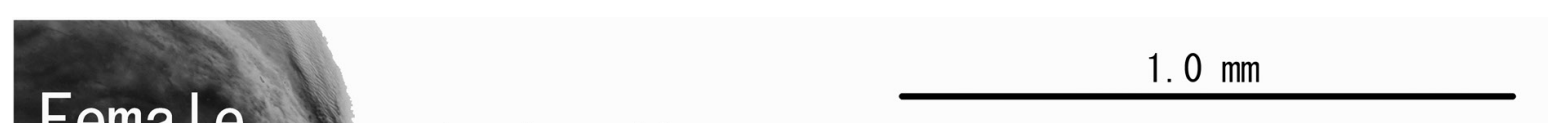
ovipositor (F)
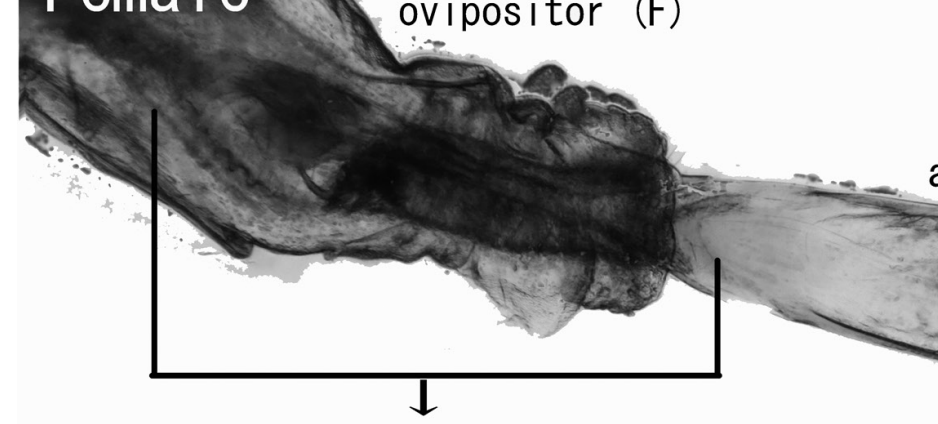
leg.”; 3 우 (EUMJ), "Nusa Dua, Bali Indonesia 5-I-2000 H. Yoshitomi leg."; 2 우 (EUMJ), ditto but collector "M. Satô"; 1 + (EUMJ), ditto but collector "N. Ohbayashi".

\section{Description}

\section{Male}

Body oblong, shining, densely covered with short black setae. Coloration of body black with strong bluish luster on head, pronotum and elytra; labrum, maxillary palpomere III and tarsi brown; antennomeres I-III and maxillary palpomeres I-II yellowish-orange.

Head slightly narrower than pronotum, densely punctate; vertex flat dorsally; eyes large, strongly prominent; HL/HW 0.73-0.82 (0.78). Antennae (Fig. 7A) stout; antennomere III (Fig. 8A) oval, deeply concave in dorso-mesal portion, $\mathrm{L} / \mathrm{W}=0.95$; approximate ratio of each antennomeres $(\mathrm{n}=1)$ as 8.0 : $1.0: 6.0: 2.2: 2.0: 1.8: 1.8: 1.8: 2.0: 1.8: 2.8$. Pronotum quadrate, widest near anterior margin, rounded and obscure in antero- and postero-lateral angles, punctate as in head; PW/PL 1.15-1.44 (1.26). Scutellum semicircular, covered with very fine punctures. Elytra oblong, subparallel-sided near base to apical 1/4; EL/EW 1.33-1.71 (1.50); EL/PL 2.50-2.89 (2.65); EW/PW 1.30-1.52 (1.41); TL/EW 2.33-2.93 (2.58). Legs relatively long and slender. Caudal margin of tergite VIII shallowly concave.

Aedeagus (Fig. 9A) about $1.12 \mathrm{~mm}$, round at aedeagal apex. Gonoporal piece (Fig. 3A) straight, rather wide, $0.61 \mathrm{~mm}$ in GL; GL/AL 2.39. Ligula (Fig. 3A) short, curved in basal 1/3; LL $0.23 \mathrm{~mm}$; LW $0.06 \mathrm{~mm} ; \mathrm{LL} / \mathrm{LW} 3.73$; GL/LL 2.68.

\section{Female}

Similar to male in general appearance; HL/HW 0.71-0.79 (0.76); PW/PL 1.20-1.36 (1.27); EL/ EW 1.53-1.68 (1.59); EL/PL 2.65-2.93 (2.82); EW/PW 1.31-1.44 (1.39); TL/EW 2.60-2.82 (2.68). Antennae (Fig. 7A) stout; antennomere III short, $\mathrm{L} / \mathrm{W}=1.88$; approximate ratio of each antennomeres $(\mathrm{n}=1)$ as $8.8: 1.0: 4.2: 2.4: 2.2: 2.2: 2.0: 1.8: 2.0: 2.0: 3.0$.

\section{Measurements}

Male (n= 7): TL 4.10-4.46 (4.27) mm; HL 0.80-0.90 (0.85) mm; HW 1.00-1.20 (1.10) mm; PW 1.081.30 (1.18) mm; PL 0.90-1.00 (0.94) mm; EL 2.40-2.60 (2.48) mm; EW 1.40-1.82 (1.67) mm. Female $(\mathrm{n}=7)$ : TL 4.35-4.75 (4.61) mm; HL 0.85-0.95 (0.90) mm; HW 1.15-1.25 (1.20) mm; PW 1.20-1.30 (1.24) mm; PL 0.90-1.00 (0.97) mm; EL 2.60-2.80 (2.73) mm; EW 1.60-1.80 (1.72) mm.

\section{Distribution}

Only known from the type locality, Indonesia (Bali Isl.).

\section{Remarks}

This species is similar to Laius flavicornis (Fabricius, 1801) and L. sabangensis Wittmer, 1985 in the shape of male antennomere III, and differs from them in the shape of endophallic sclerites and smaller body length.

\section{Biological notes}

This species was collected together with Laius pankowi Wittmer, 1999 and L. satoi Yoshitomi, 2008 at the same locality. Based on the data of Yoshitomi (2008) and the present study, Laius baliensis sp. nov. was collected in January and August, L. pankowi in January and December, and L. satoi in January, April, and August. 
Laius andamanensis sp. nov.

urn:1sid:zoobank.org:act:AB272070-CAEA-4964-8CFD-A219E93222F1

Figs 3B, 5C-D, 7B, 8B, 9B, 12

\section{Diagnosis}

Male antennomere III distinctly projecting laterally, shallowly concave in dorso-mesal portion, L/W = 0.70; aedeagal apex concave; gonoporal piece straight and slender; ligula long and slightly curved; additional sclerite absent.

\section{Etymology}

After the type locality, Andaman Islands, India.

\section{Type material}

\section{Holotype}

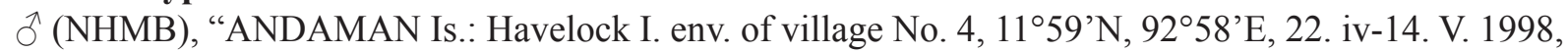
Karel \& Simon Majer leg.”.

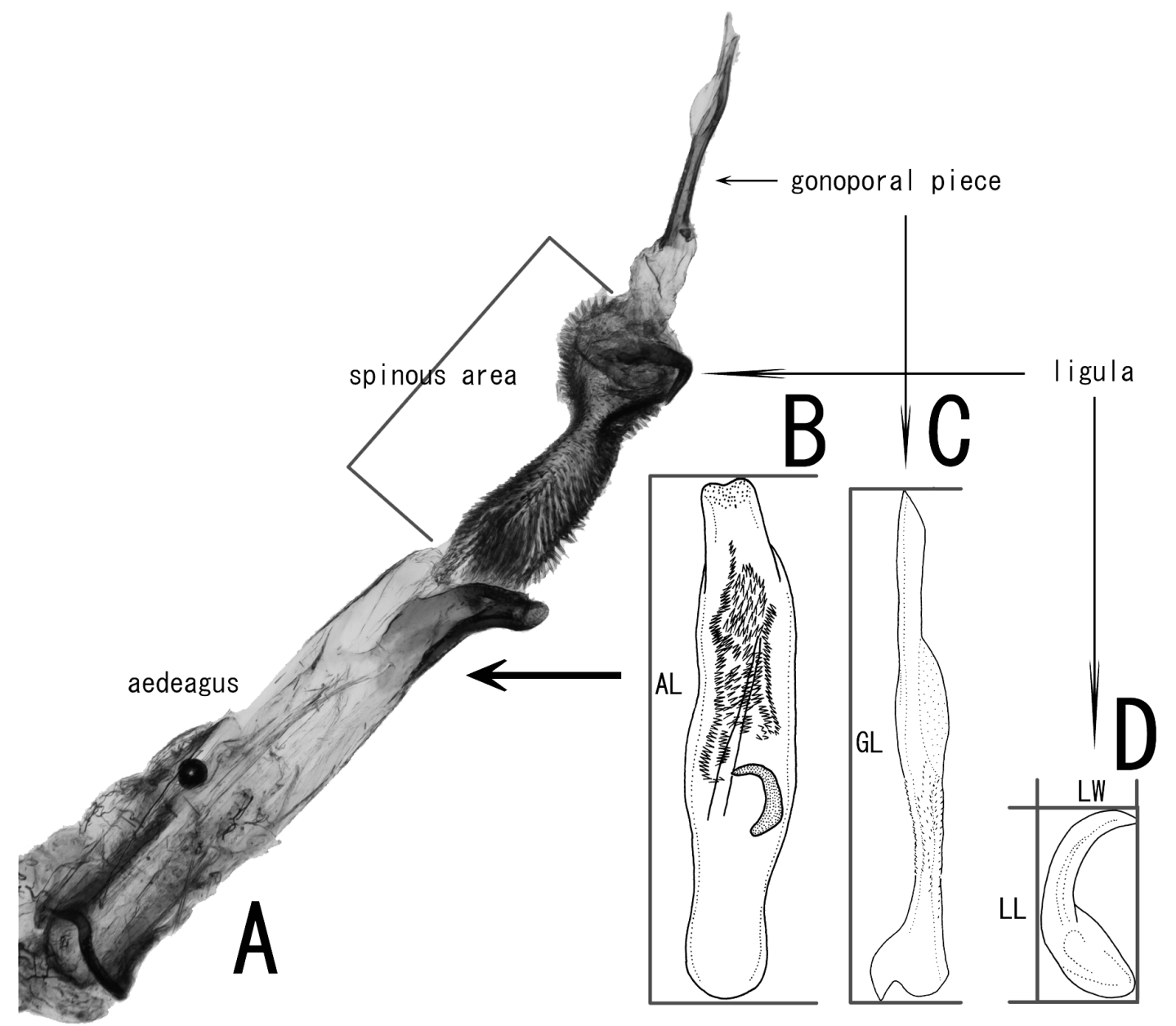

Fig. 2. Aedeagus of Laius lutaoensis Yoshitomi \& Lee, 2010, showing the terminology and abbreviations for measurement of endophallic structures. A. Aedeagus with extracted endophallus. B. Aedeagus with holding endophallus (from Yoshitomi \& Lee 2010). C. Gonoporal piece. D. Ligula. 


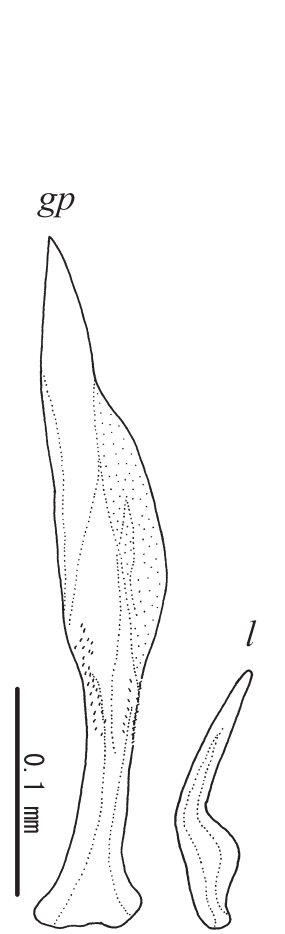

A

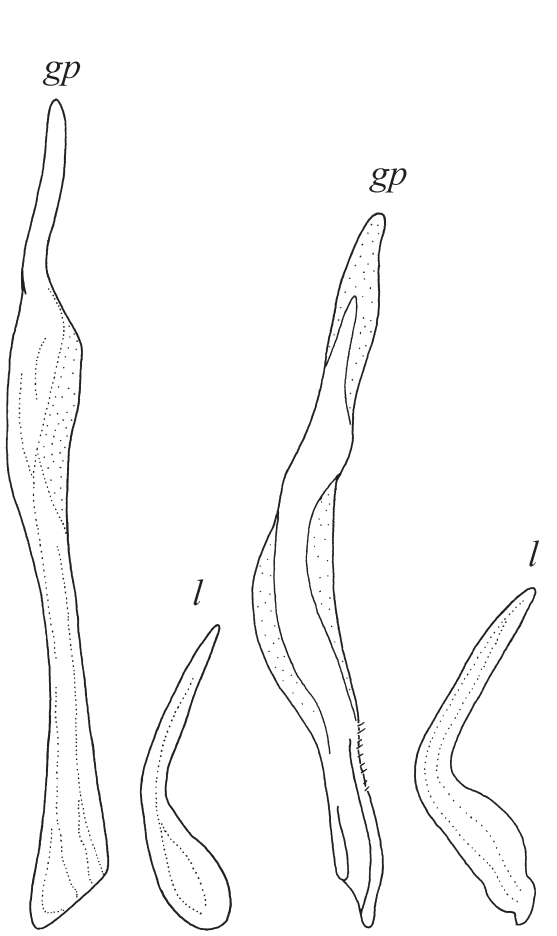

$\mathrm{B}$

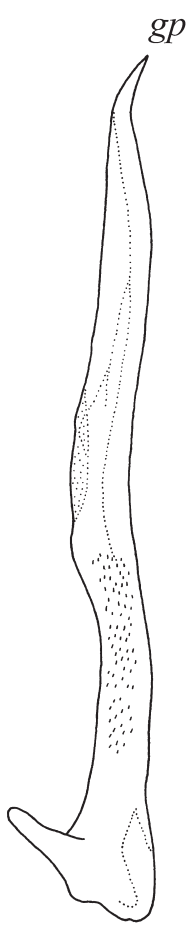

C

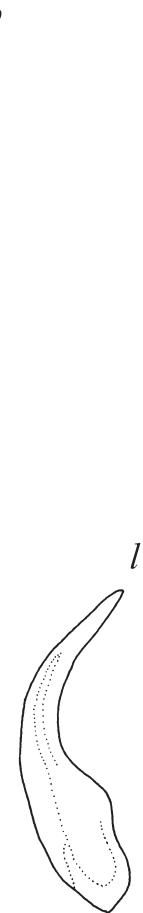

D

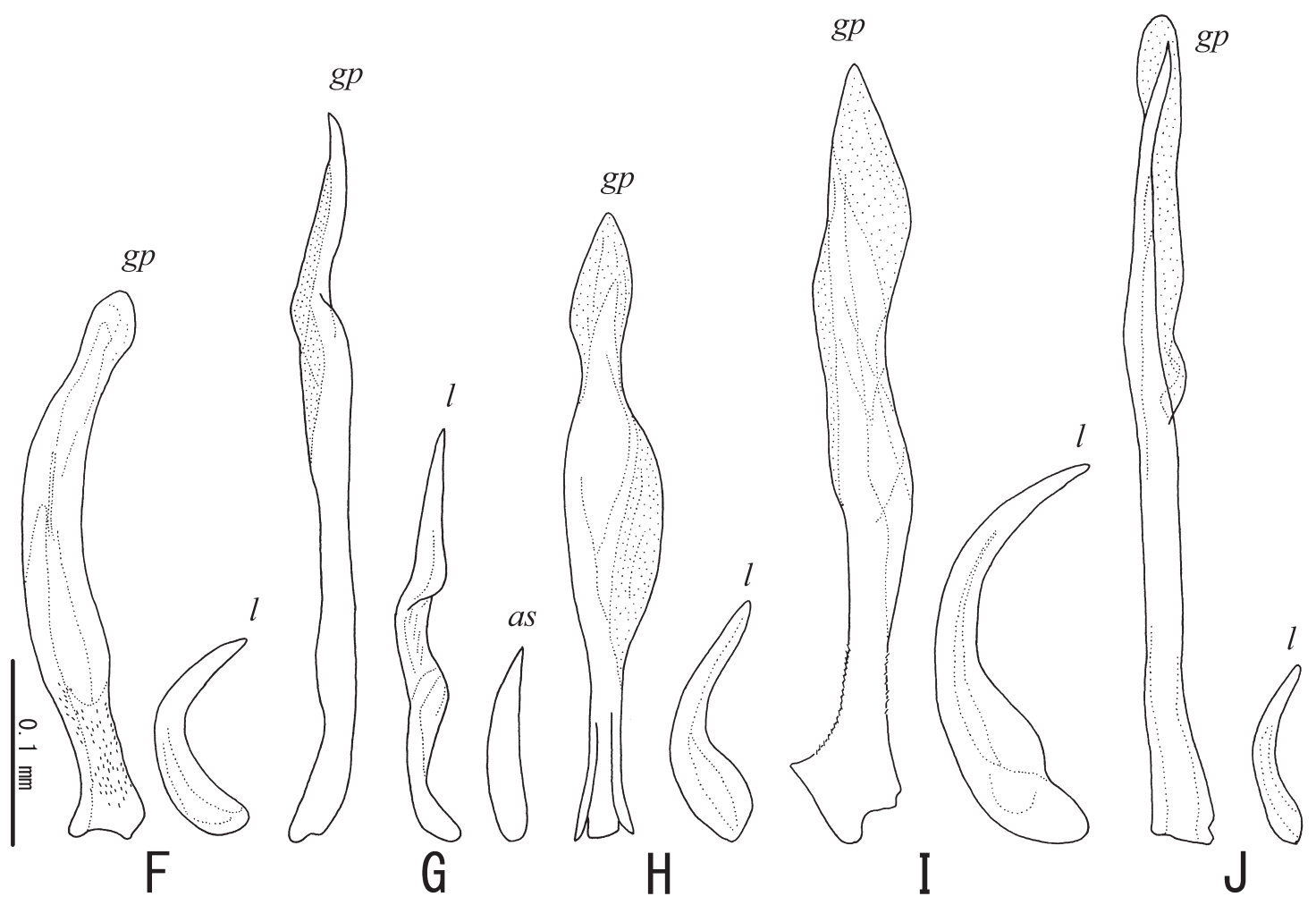

Fig. 3. Endophallic sclerites of Laius spp. A. Laius baliensis sp. nov. (paratype). B. L. andamanensis sp. nov. (paratype). C. L. rodriguesensis sp. nov. (paratype). D. L. maai Wittmer, 1973 (paratype). E. L. marchei Pic, 1922. F. L. politus Fairmaire, 1880. G. L. sabangensis Wittmer, 1985 (paratype). H. L. sericatus Champion, 1924. I. L. submariniformis Wittmer, 1985 (paratype). J. L. asahinai Nakane, 1955. 

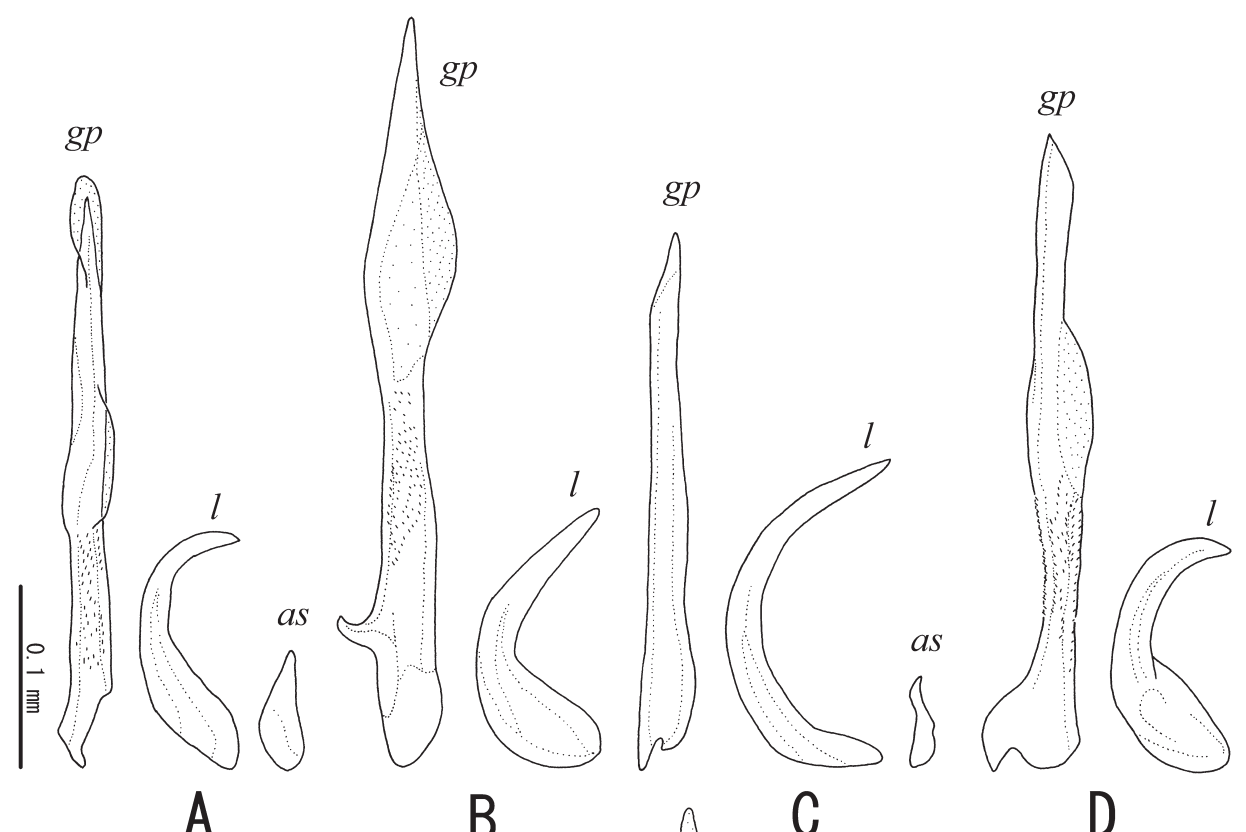

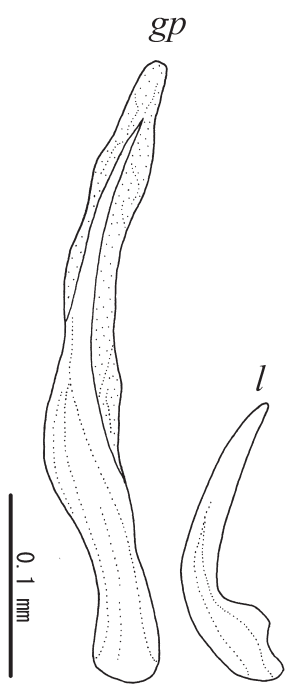

$\mathrm{E}$

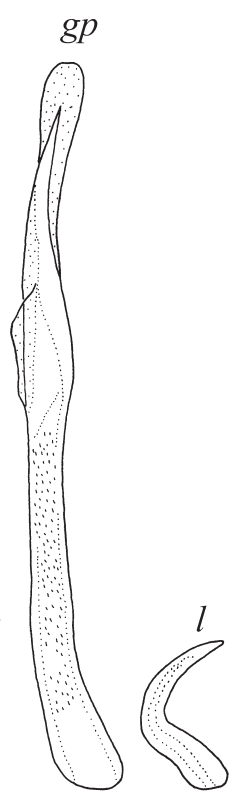

$\mathrm{F}$

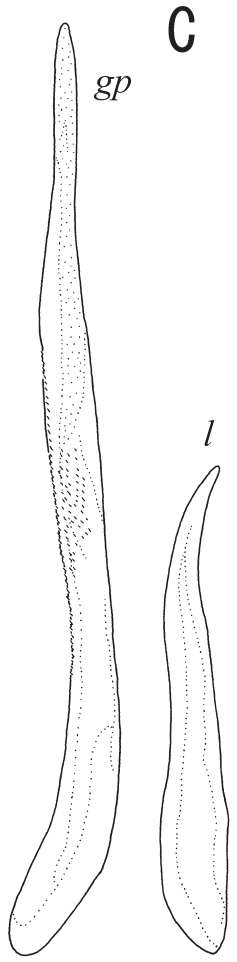

G

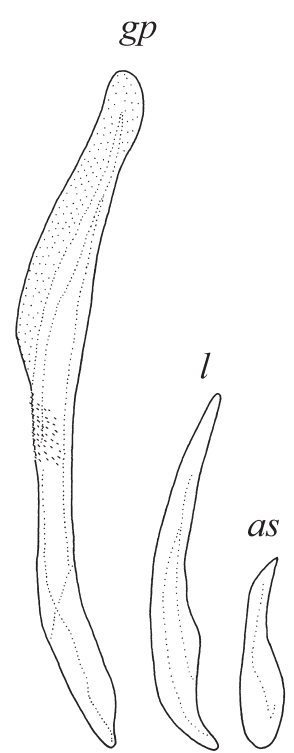

$\mathrm{H}$

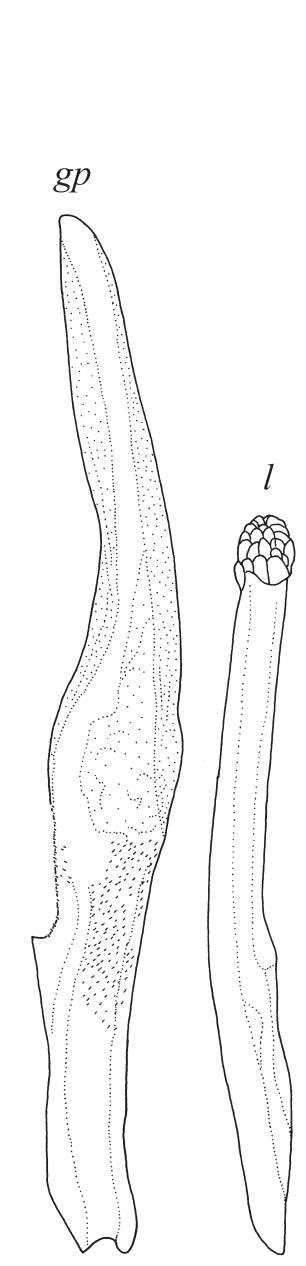

I

Fig. 4. Endophallic sclerites of Laius spp. A. Laius etsukoae Satô, Yoshitomi \& Ohbayashi, 2006 (paratype). B. L. Alavicornis (Fabricius, 1801). C. L. keiichii Satô, Yoshitomi \& Ohbayashi, 2006 (paratype). D. L. lutaoensis Yoshitomi \& Lee, 2010 (paratype). E. L. madli Yoshitomi, 2010 (paratype). F. L. miyamotoi Nakane, 1955. G. L. satoi Yoshitomi, 2008. H. L. taiwanus Yoshitomi \& Lee, 2010 (paratype). I. L. pankowi Wittmer, 1999. 
Paratypes

$22 \widehat{\partial} \widehat{\partial}, 10$ 우 (NHMB, EUMJ), same data as for the holotype.

\section{Description}

Male

Body oblong, shining, densely covered with short black setae. Coloration of body black with dully bluish luster on elytra; labrum, maxillary palpomere I, and tarsi brown; antennomeres I-III yellowish-orange.

Head narrower than pronotum, very densely punctate; vertex slightly convex dorsally; eyes relatively large, prominent; HL/HW 0.76-0.90 (0.81). Antennae (Fig. 7B) long; antennomere III (Fig. 8B) transversal trapezoidal, distinctly projecting laterally, shallowly concave in dorso-mesal portion, $\mathrm{L} / \mathrm{W}$ $=0.70$; approximate ratio of each antennomeres $(\mathrm{n}=1)$ as $8.3: 1.0: 5.0: 2.0: 2.2: 2.0: 2.0: 2.0:$ $2.0: 2.0: 3.5$. Pronotum quadrate, widest near anterior margin, rounded and obscure in antero- and postero-lateral angles, punctate as in head; PW/PL 1.18-1.33 (1.24). Scutellum semicircular, covered with fine punctures. Elytra oblong, widest at apical 1/4; sides gradually expanded postero-laterally; EL/ EW 1.36-1.55 (1.45); EL/PL 2.52-2.88 (2.67); EW/PW 1.38-1.55 (1.48); TL/EW 2.30-2.56 (2.44). Legs relatively long and slender; fore tibia (Fig. 12A) enlarged, deeply excavate. Caudal margin of tergite VII shallowly concave.

Aedeagus (Fig. 9B) about $1.13 \mathrm{~mm}$, concave at aedeagal apex (Fig. 12C). Gonoporal piece (Fig. 3B) straight, slender, $0.74 \mathrm{~mm}$ in GL; GL/AL 2.39. Ligula (Fig. 3B) relatively long, slightly curved; LL $0.27 \mathrm{~mm}$; LW 0.11 mm; LL/LW 2.58; GL/LL 2.71.

\section{Female}

Similar to male in general appearance. HL/HW 0.75-0.80 (0.79); PW/PL 1.18-1.30 (1.23); EL/ EW 1.36-1.48 (1.41); EL/PL 2.44-2.73 (2.60); EW/PW 1.45-1.58 (1.51); TL/EW 2.30-2.43 (2.36). Antennae (Fig. 7B) long; antennomere III (Fig. 12B) distinctly expanded laterally, shallowly concave in dorsal portion, $\mathrm{L} / \mathrm{W}=2.42$; approximate ratio of each antennomeres $(\mathrm{n}=1)$ as $8.0: 1.0: 4.4: 2.2: 2.4$ : $2.2: 2.2: 2.0: 2.0: 2.0: 3.6$

\section{Measurements}

Male (n=20): TL 5.52-6.33 (5.79) mm; HL 1.00-1.15 (1.05) mm; HW 1.22-1.38 (1.30) mm; PW 1.481.75 (1.61) mm; PL 1.20-1.48 (1.29) mm; EL 3.28-3.75 (3.45) mm; EW 2.18-2.70 (2.38) mm. Female $(\mathrm{n}=6)$ : TL 4.43-4.96 (4.67) mm; HL 0.75-0.85 (0.81) mm; HW 1.00-1.08 (1.03) mm; PW 1.20-1.42 (1.32) mm; PL 1.00-1.20 (1.07) mm; EL 2.60-3.00 (2.79) mm; EW 1.85-2.15 (1.98) mm.

\section{Distribution}

Only known from the type locality, India (Andaman Islands).

\section{Remarks}

This is distinct species in having transversal antennomere III of male.

Laius rodriguesensis sp. nov. urn:1sid:zoobank.org:act:5ACC9D36-0154-4FE3-9D27-D5AAF61A9143

Figs 3C, 5E-F, 7C, 8C, 9C, 10, 11

\section{Diagnosis}

Male antennomere III transverse, deeply concave in dorso-mesal portion, $\mathrm{L} / \mathrm{W}=0.74$; aedeagal apex rounded; gonoporal piece slightly curved; ligula strongly curved; additional sclerite absent; coloration of female antennomeres I-III black or dark brown. 


\section{Etymology}

After the type locality, Rodrigues Island, Republic of Mauritius.

\section{Type material}

\section{Holotype}

$\widehat{\jmath}$ (NMW), "RODRIGUES Bucht zw. Trou d'Argent und Anse Bouteille intertidal zone 12.-13.4.11, leg. M. Madl".
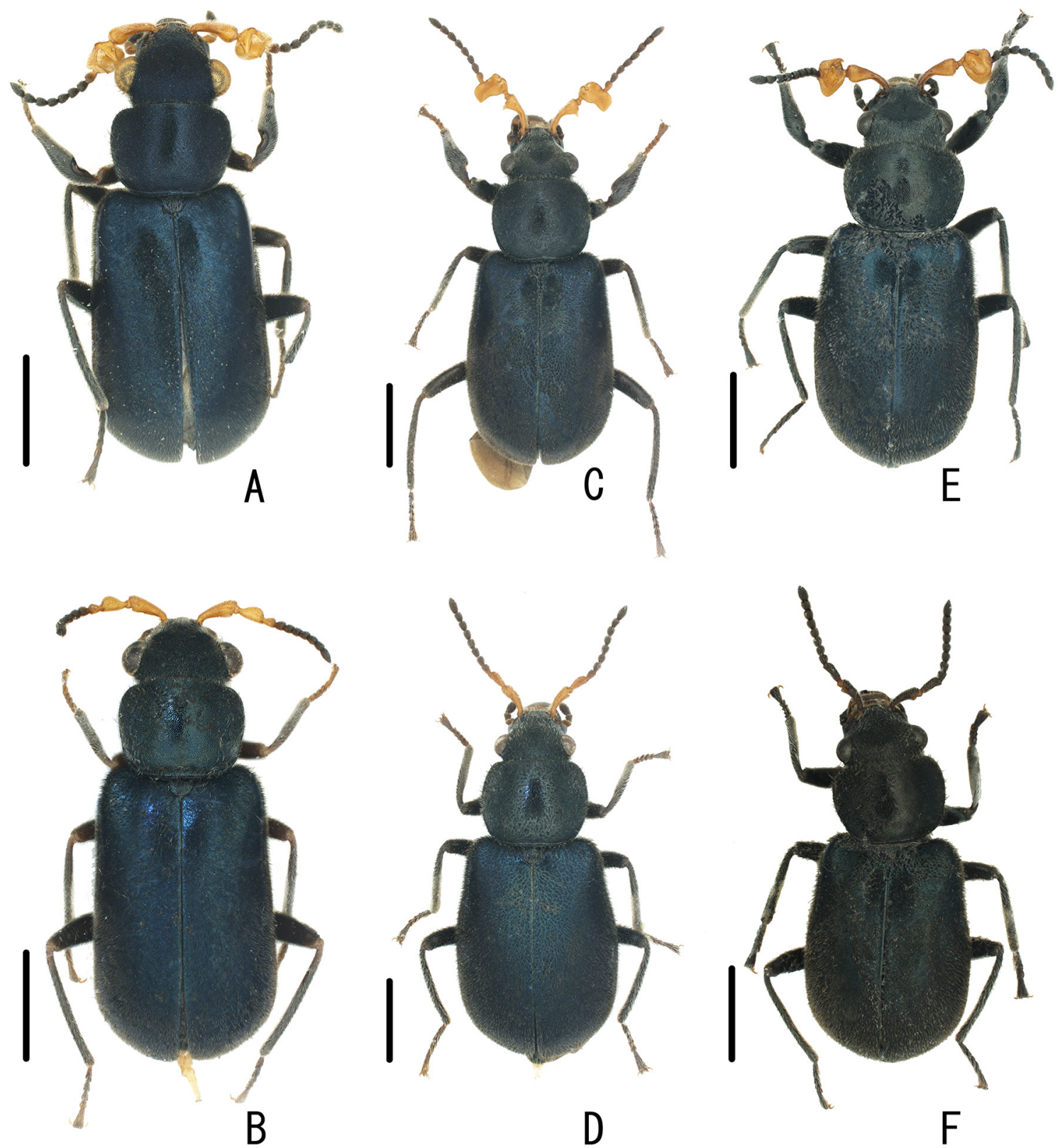

Fig. 5. Dorsal habitus of Laius spp. of $\hat{O}$ (holotypes A, C, E) and $q$ 우 (paratypes B, D, F). A-B. Laius baliensis sp. nov. C-D. L. andamanensis sp. nov. E-F. L. rodriguesensis sp. nov. Scale bars $=1.0 \mathrm{~mm}$. 


\section{Paratypes}

3 + 9 (NMW), same data as for the holotype; 1 (NMW), "MAURITIUS Rodrigues, Latanier Rivière Cascade Victoire 7.5.08. leg. M. Madl"; 2 ㅇ (NMW), "MAURITIUS Rodrigues Anse Fumier 2.4.09, leg. M. Madl"; 4 ふึग, 11 우 (NMW, EUMJ), ditto but "2.5.08".

\section{Description}

\section{Male}

Body oblong, shining, densely covered with short black setae. Coloration of body black with dully bluish luster on elytra; antennomeres I-III yellowish-orange, but anterior margin of antennomere I infuscate.

Head narrower than pronotum, very densely punctate; vertex slightly depressed dorsally; eyes relatively large, prominent; HL/HW 0.72-0.89 (0.78). Antennae (Fig. 7C) long; antennomere III (Fig. 8C) transverse, deeply concave in dorso-mesal portion, $\mathrm{L} / \mathrm{W}=0.74$; approximate ratio of each antennomeres $(\mathrm{n}=1)$ as $11.2: 1.0: 6.8: 3.2: 2.6: 2.2: 2.2: 2.4: 2.0: 2.2: 4.0$. Pronotum quadrate, widest near anterior margin, rounded and obscure in antero- and postero-lateral angles; punctate as in head; PW/PL 1.33-1.49 (1.39). Scutellum semicircular, finely punctate. Elytra oblong, widest at apical 1/3; sides weakly expanded postero-laterally; EL/EW 1.31-1.50 (1.43); EL/PL 2.62-2.76 (2.69); EW/PW 1.31-1.42 (1.36); TL/EW 2.18-2.52 (2.42). Legs relatively long and slender. Caudal margin of tergite VII shallowly concave. Aedeagus (Fig. 9C) about $1.17 \mathrm{~mm}$, round at aedeagal apex. Gonoporal piece (Fig. 3C) slightly curved, $0.63 \mathrm{~mm}$ in GL; GL/AL 1.81. Ligula (Fig. 3C) long, strongly curved in half; LL $0.30 \mathrm{~mm}$; LW $0.11 \mathrm{~mm}$; LL/LW 2.84; GL/LL 2.11.

\section{Female}

Similar to male in general appearance; bluish luster on elytra weak; coloration of antennomeres I-III black or dark brown; HL/HW 0.74-0.95 (0.84); PW/PL 1.32-1.42 (1.37); EL/EW 1.29-1.47 (1.39); EL/PL 2.47-2.86 (2.68); EW/PW 1.30-1.48 (1.41); TL/EW 2.25-2.47 (2.39). Antennae (Fig. 7C) long; antennomere III short, $\mathrm{L} / \mathrm{W}=2.16$; approximate ratio of each antennomeres $(\mathrm{n}=1)$ as $8.6: 1.0: 4.4$ : $2.0: 2.0: 1.8: 1.8: 2.0: 2.0: 2.0: 3.8$.

\section{Measurements}

Male (n= 5): TL 5.50-5.75 (5.64) mm; HL 1.00-1.20 (1.08) mm; HW 1.30-1.45 (1.38) mm; PW 1.601.83 (1.72) mm; PL 1.20-1.30 (1.24) mm; EL 3.20-3.40 (3.32) mm; EW 2.20-2.60 (2.33) mm. Female $(\mathrm{n}=10)$ : TL 4.10-4.68 (4.41) mm; HL 0.80-1.00 (0.88) mm; HW 1.00-1.10 (1.05) mm; PW 1.20-1.38 (1.32) mm; PL 0.90-1.00 (0.96) mm; EL 2.35-2.80 (2.57) mm; EW 1.70-2.00 (1.85) mm.

\section{Larvae}

Coloration of head capsule and urogomphi dark brown; thoracic and abdominal segments and legs cream. Head capsule (Fig. 10B) suboblong, L/W = 1.3; four pairs of stemmata situated near anterolateral corners. Antennae missing. Labrum (Fig. 11B) with a pair of long lateral and five pairs of short mesal setae in anterior portion; anterior margin of epipharynx bearing short stout setae. Maxillae (Fig. 11A) bearing long setae in antero-lateral portion. Mandibles (Fig. 11C) with two apical teeth; mola with two short teeth. Labium (Figs 10C, 11A) relatively wide, with 2-segmented palpi. Thorax weakly sclerotized, widest at posterior part of metathorax; pronotum with two pairs of longitudinal sclerotized bands in mesal portion, bearing short setae in lateral and mesal portions. Abdomen (Fig. 10A) weakly sclerotized, subparallel-sided, bearing short setae in lateral and postero-mesal portions. Urogomphi (Fig. 10D-F) subparallel-sided, closely covered with long setae, simply bifurcate in apical 1/2, upturned in apical portions, pointed at apices, with some very long hairy setae in apical parts. Legs relatively long.

\section{Additional material examined}

2 Larvae (NMW, in ethanol), "MAURITIUS Rodrigues Anse Fumier 10.05.2008, leg. M. Madl”. 


\section{Distribution}

Only known from the type locality, Republic of Mauritius (Rodrigues Isl.).

\section{Remarks}

This species is closely related to Laius madli Yoshitomi, 2010 known from Mauritius, but differs from the latter in the following characteristics: 1) ligula strongly curved in middle portion (rather weakly curved

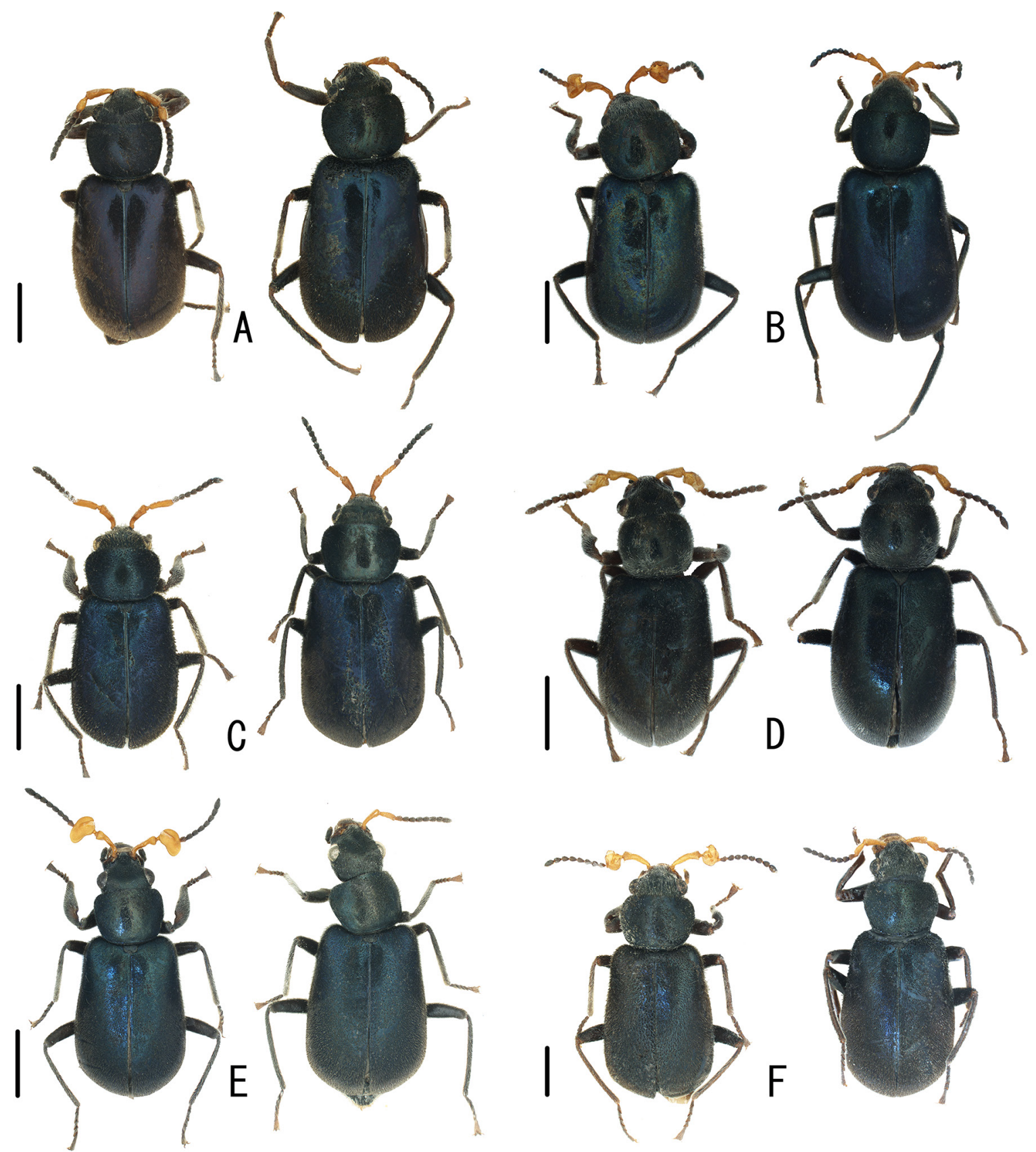

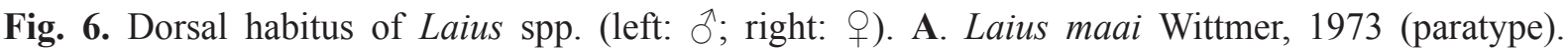
B. L. marchei Pic, 1922. C. L. politus Fairmaire, 1880. D. L. sabangensis Wittmer, 1985 (paratype). E. L. sericatus Champion, 1924. F. L. submariniformis Wittmer, 1985 (paratype). Scale bars $=1.0 \mathrm{~mm}$. 
basally in madli); 2) male antennomere III projecting laterally, transverse, $\mathrm{L} / \mathrm{W}=0.7$ (antennomere III slightly projecting laterally, $\mathrm{L} / \mathrm{W}=0.9$ in madli).

The larvae of two Japanese species, L. asahinai Nakane, 1955 and L. miyamotoi Nakane, 1955, were described by Satô (1964) and Asano \& Kojima (2009). The larva of L. rodrigesensis sp. nov. is similar to those of the aforementioned species, and differs from them in that the teeth of the mola are rather short, and that the head is narrower.

Laius maai Wittmer, 1973

Figs 3D, 6A, 7D, 8D, 9D

Laius maai Wittmer, 1973: 336.

\section{Type material}

Paratypes $6 \hat{\jmath} \widehat{\partial}, 2$ 우, (BPBM), "New Guinea: NE Sarmi, W Hollandia”, “20.-23.7.1959 T. C. Maa”.

\section{Redescription}

Male

HL/HW 0.82; PW/PL 1.36; EL/EW 1.51; EL/PL 2.95; EW/PW 1.43; TL/EW 2.46. Antennae (Fig. 7D) long; antennomere III (Fig. 8D) oblong, widened in basal 1/3, L/W $=2.08$; approximate ratio of each antennomeres $(n=1)$ as $7.4: 1.0: 3.6: 2.1: 1.9: 1.9: 1.7: 1.6: 1.7: 1.9: 3.1$. Caudal margin of tergite VII concave. Aedeagus (Fig. 9D) about $1.32 \mathrm{~mm}$, concave at aedeagal apex. Gonoporal piece (Fig. 3D) straight, slender, with long and straight basal projection, $0.77 \mathrm{~mm}$ in GL; GL/AL 2.01. Ligula (Fig. 3D) relatively long, slightly curved; LL 0.29 mm; LW 0.09 mm; LL/LW 3.06; GL/LL 2.65.

\section{Female}

HL/HW 0.78-0.81 (0.80); PW/PL 1.31-1.37 (1.33); EL/EW 1.42-1.66 (1.50); EL/PL 2.70-2.96 (2.83); EW/PW 1.35-1.46 (1.41); TL/EW 2.39-2.67 (2.48). Antennae (Fig. 7D) long; antennomere III short, $\mathrm{L} / \mathrm{W}=1.88$; approximate ratio of each antennomeres $(\mathrm{n}=1)$ as $11.3: 1.0: 5.3: 2.8: 3.0: 3.0: 3.0: 2.5$ : $2.8: 2.5: 4.5$.

\section{Measurements}

Male (n=1): TL 5.05 mm; HL 0.90 mm; HW $1.10 \mathrm{~mm}$; PW $1.43 \mathrm{~mm}$; PL $1.05 \mathrm{~mm}$; EL $3.10 \mathrm{~mm}$; EW $2.05 \mathrm{~mm}$. Female ( $=4)$ : TL 4.50-4.68 (4.58) mm; HL 0.80-0.85 (0.82) mm; HW 1.00-1.05 (1.03) mm; PW 1.30-1.33 (1.31) mm; PL 0.95-1.00 (0.98) mm; EL 2.70-2.90 (2.78) mm; EW 1.75-1.90 (1.85) mm.

\section{Distribution}

New Guinea.

Laius marchei Pic, 1922

Figs 3E, 6B, 7E, 8E, 9E

Laius marchei Pic, 1922: 158.

Rotamalachius esakii Kôno, 1937 in Kôno \& Esaki 1937: 121. Synonymised by Nakane (1955).

Laius marchei - Wittmer 1958: 72 [redescription]. — Satô et al. 2006: 344.

\section{Material examined}

$56 \widehat{\jmath} \widehat{\jmath}, 14$ 우 (EUMJ), “(MARIANA Is.) Teteto Beach Rota I. 22. Jan. 1980 M. Tomokuni”; 1 ㅇ (EUMJ), "Is. Rota mariana VIII. 8. 1940 D. Matsusita", "Laius marchei Pic det. W. Wittmer"; 1 స 
(EUMJ), "(Rota Tatochio) South Sea Islands of Nippon: 8-VIII. 1940 Coll. Dengo Matsusita"; 2 ðð, 1 (BPBM), “Tumon Bay, Guam 24 June 1969”, “A. A. LaPlante Collection BISHOP Museum Acc. \#1977.84".

\section{Redescription}

Male

HL/HW 0.71-0.93 (0.79); PW/PL 1.21-1.36 (1.28); EL/EW 1.28-1.48 (1.42); EL/PL 2.42-2.86 (2.68); EW/PW 1.41-1.58 (1.48); TL/EW 2.25-2.44 (2.36). Antennae (Fig. 7E) long; antennomere I long and curved laterally, with small projection in lateral margin; antennomere III (Fig. 8E) trapezoidal, deeply concave in dorso-mesal portion, $\mathrm{L} / \mathrm{W}=0.76$; approximate ratio of each antennomeres $(\mathrm{n}=1)$ as 11.8 : $1.0: 7.0: 2.6: 2.0: 2.2: 2.2: 2.4: 2.4: 2.4: 3.8$. Caudal margin of tergite VII concave. Aedeagus

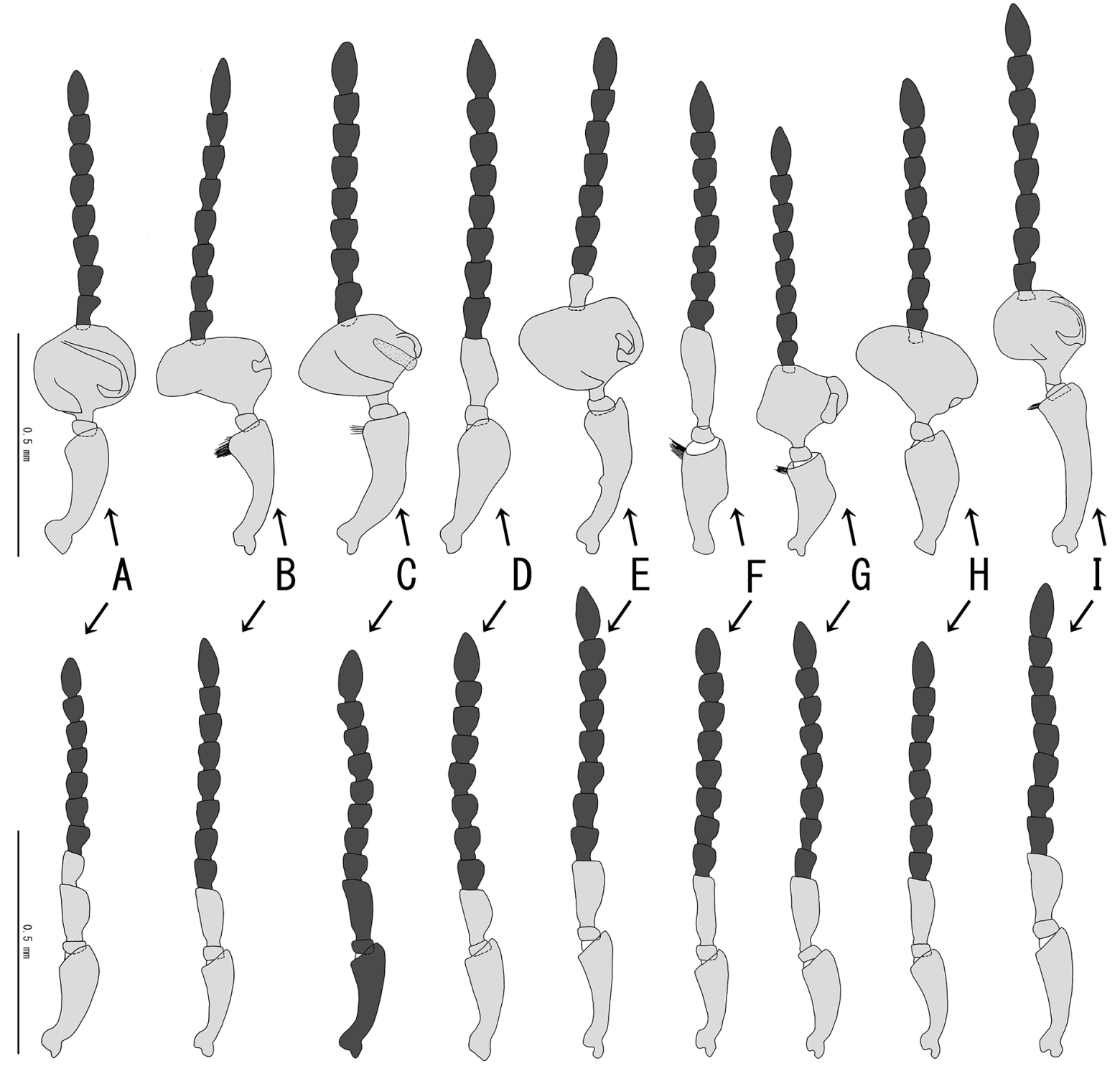

Fig. 7. Left antennae of Laius spp. (above: male; below: female). A. Laius baliensis sp. nov. (paratype). B. L. andamanensis sp. nov. (paratype). C. L. rodriguesensis sp. nov. (paratype). D. L. maai Wittmer, 1973 (paratype). E. L. marchei Pic, 1922. F. L. politus Fairmaire, 1880. G. L. sabangensis Wittmer, 1985 (paratype). H. L. sericatus Champion, 1924. I. L. submariniformis Wittmer, 1985 (paratype). 
(Fig. 9E) about $1.28 \mathrm{~mm}$, concave at aedeagal apex. Gonoporal piece (Fig. 3E) straight, slender, with long and straight basal projection, $0.80 \mathrm{~mm}$ in GL; GL/AL 1.91. Ligula (Fig. 3E) long and slender, strongly curved in apical half; LL 0.33 mm; LW 0.17 mm; LL/LW 2.81; GL/LL 2.44.

\section{Female}

HL/HW 0.72-0.87 (0.80); PW/PL 1.13-1.32 (1.24); EL/EW 1.43-1.55 (1.46); EL/PL 2.64-2.96 (2.82); EW/PW 1.52-1.67 (1.57); TL/EW 2.34-2.45 (2.38). Antennae (Fig. 7E) long; antennomere III short, $\mathrm{L} / \mathrm{W}=2.25$; approximate ratio of each antennomeres $(\mathrm{n}=1)$ as $9.4: 1.0: 5.0: 2.4: 2.8: 2.4: 2.4: 2.4$ : $2.4: 2.4: 4.0$.

\section{Measurements}

Male ( $\mathrm{n}=15)$ : TL 4.90-6.75 (5.36) mm; HL 0.80-1.40 (0.94) mm; HW 1.10-1.50 (1.19) mm; PW 1.351.90 (1.53) mm; PL 1.10-1.50 (1.20) mm; EL 2.90-3.85 (3.21) mm; EW 2.10-3.00 (2.27) mm. Female $(\mathrm{n}=6)$ : TL 5.15-5.73 (5.37) mm; HL 0.85-1.00 (0.92) mm; HW 1.10-1.25 (1.15) mm; PW 1.35-1.50 (1.44) mm; PL 1.10-1.30 (1.17) mm; EL 3.15-3.43 (3.28) mm; EW 2.20-2.40 (2.25) mm.

\section{Distribution}

Mariana Isls. (Rota, Guam).

\section{Remarks}

This is the first record of this species from Guam, Mariana Islands.

Laius politus Fairmaire, 1880

Figs 3F, 6C, 7F, 8F, 9F

Laius politus Fairmaire, 1880: 246.

Nossibeus politus - Evers 1994: 173 [see page 3].

\section{Material examined}

$18 \widehat{\jmath} \widehat{\jmath}, 40$ 우 (NHMB, EUMJ), “MADAGASCAR Katsepy (Majunga) 24-31. xii. 1977 P. Pacholátko leg".

\section{Redescription}

\section{Male}

HL/HW 0.69-0.94 (0.80); PW/PL 1.33-1.57 (1.43); EL/EW 1.39-1.52 (1.46); EL/PL 2.82-3.40 (3.05); EW/PW 1.37-1.59 (1.46); TL/EW 2.24-2.51 (2.36). Antennae (Fig. 7F) long; antennomere I stout; antennomere III (Fig. 8F) elongate, widened in apical portion, $\mathrm{L} / \mathrm{W}=3.08$; approximate ratio of each antennomeres $(n=1)$ as $8.4: 1.0: 7.4: 2.2: 2.0: 2.0: 2.2: 2.0: 2.2: 2.0: 3.8$. Caudal margin of tergite VII shallowly concave. Aedeagus about $1.18 \mathrm{~mm}$, round at aedeagal apex. Gonoporal piece (Fig. 3F) curved, rather wide, $0.54 \mathrm{~mm}$ in GL; GL/AL 2.45. Ligula (Fig. 3F) short, evenly curved; LL $0.19 \mathrm{~mm}$; LW 0.09 mm; LL/LW 2.13; GL/LL 2.88.

\section{Female}

HL/HW 0.64-0.90 (0.79); PW/PL 1.38-1.47 (1.42); EL/EW 1.30-1.55 (1.44); EL/PL 2.80-3.10 (2.96); EW/PW 1.37-1.54 (1.45); TL/EW 2.17-2.50 (2.35). Antennae (Fig. 7F) long; antennomere III long and slender, $\mathrm{L} / \mathrm{W}=3.1$; approximate ratio of each antennomeres $(\mathrm{n}=1)$ as $8.0: 1.0: 5.2: 2.4: 2.0: 2.2$ : $2.0: 2.2: 2.2: 2.2: 3.4$. 


\section{Measurements}

Male (n = 17): TL 5.15-5.92 (5.55) mm; HL 0.90-1.13 (1.00) mm; HW 1.18-1.33 (1.25) mm; PW 1.50-1.75 (1.61) mm; PL 1.00-1.20 (1.13) mm; EL 3.10-3.62 (3.42) mm; EW 2.15-2.60 (2.35) mm. Female $(\mathrm{n}=10)$ : TL 4.05-5.00 (4.48) mm; HL 0.70-0.90 (0.81) mm; HW 0.93-1.10 (1.02) mm; PW 1.18-1.38 (1.32) mm; PL 0.85-1.00 (0.93) mm; EL 2.50-3.10 (2.75) mm; EW 1.62-2.00 (1.92) mm.

\section{Distribution}

Madagascar.

Laius sabangensis Wittmer, 1985

Figs $3 \mathrm{G}, 6 \mathrm{D}, 7 \mathrm{G}, 8 \mathrm{G}, 9 \mathrm{G}$

Laius sabangensis Wittmer, 1985: 386.
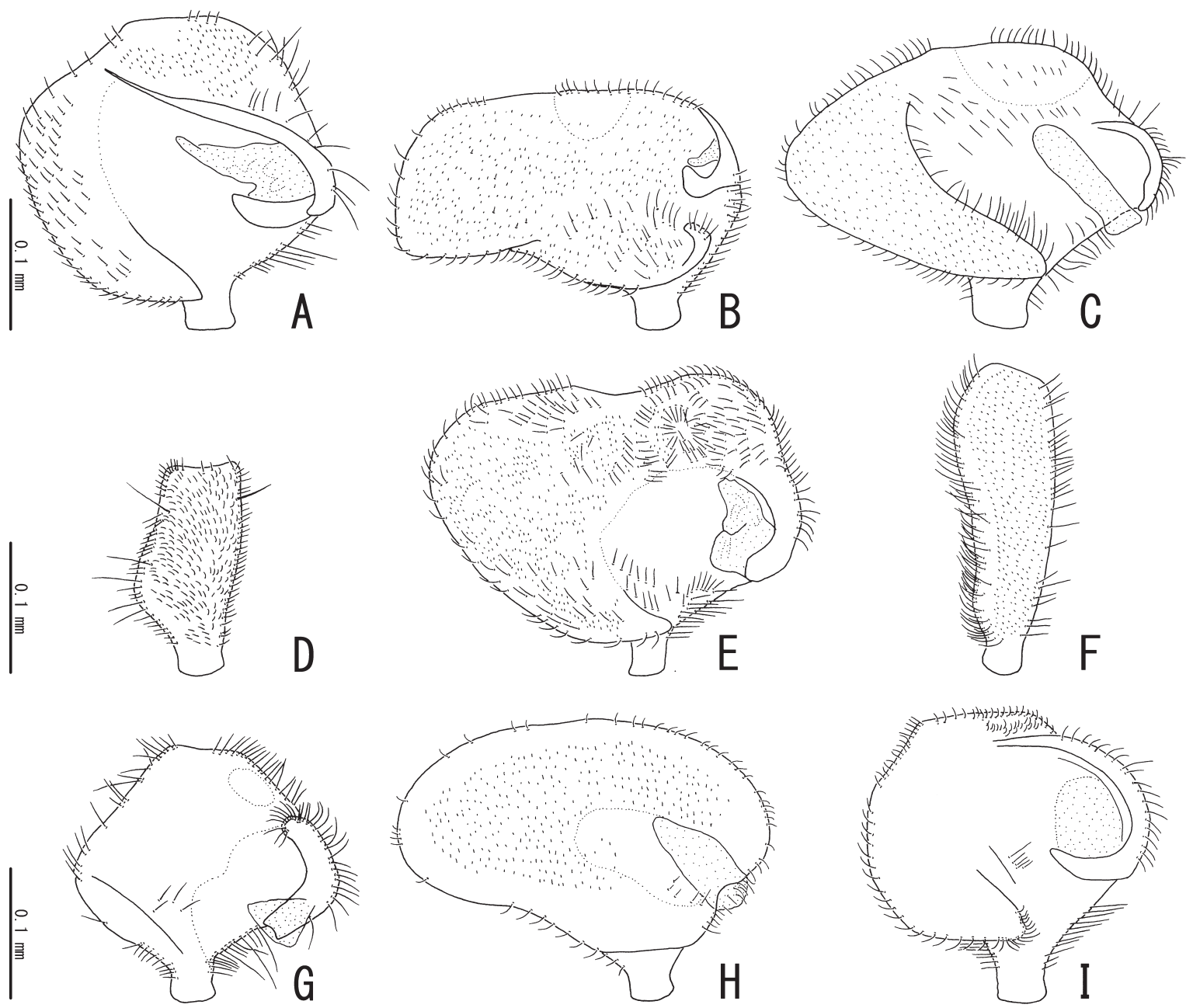

Fig. 8. Male antennomere III of Laius spp. A. Laius baliensis sp. nov. (paratype). B. L. andamanensis sp. nov. (paratype). C. L. rodriguesensis sp. nov. (paratype). D. L. maai Wittmer, 1973 (paratype). E. L. marchei Pic, 1922. F. L. politus Fairmaire, 1880. G. L. sabangensis Wittmer, 1985 (paratype). H. L. sericatus Champion, 1924. I. L. submariniformis Wittmer, 1985 (paratype). 


\section{Material examined}

Holotype, $\widehat{o}$ (EUMJ), "PHILIPPINES Sabang, N. of Mt. St. Paul, Palawan Julu, 11-13, 1977 M. Sato

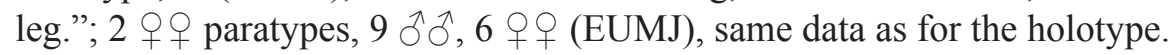

\section{Redescription}

\section{Male}

HL/HW 0.84-0.91 (0.88); PW/PL 1.10-1.22 (1.16); EL/EW 1.38-1.50 (1.43); EL/PL 2.53-2.67 (2.59); EW/PW 1.50-1.62 (1.56); TL/EW 2.37-2.60 (2.48). Antennae (Fig. 7G) stout and short; antennomere I stout; antennomere III (Fig. 8G) trapezoidal, deeply concave in dorso-mesal portion, $\mathrm{L} / \mathrm{W}=0.86$; approximate ratio of each antennomeres $(\mathrm{n}=1)$ as $6.6: 1.0: 6.0: 2.4: 2.0: 2.2: 2.0: 2.2: 2.0: 2.2$ : 3.6. Caudal margin of tergite VII straight. Aedeagus (Fig. 9G) about $0.94 \mathrm{~mm}$, concave at aedeagal apex. Gonoporal piece (Fig. 3G) straight, slender, $0.72 \mathrm{~mm}$ in GL; GL/AL 1.87. Ligula (Fig. 3G) long and winding; LL $0.41 \mathrm{~mm}$; LW 0.06 mm; LL/LW 7.30; GL/LL 1.77. Additional sclerite (Fig. 3G) present, $0.10 \mathrm{~mm}$.

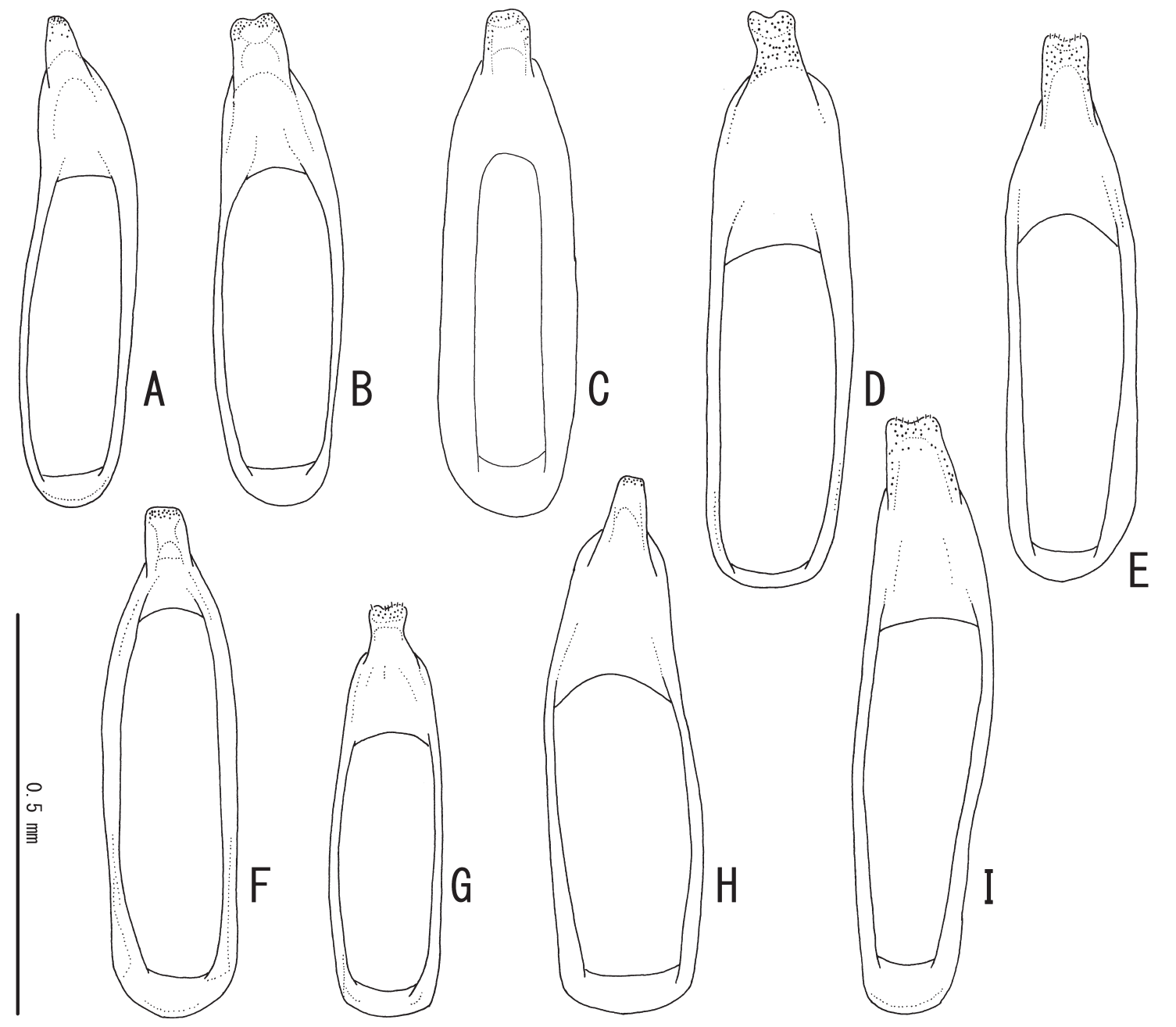

Fig. 9. Aegeagi of Laius spp. A. Laius baliensis sp. nov. (paratype). B. L. andamanensis sp. nov. (paratype). C. L. rodriguesensis sp. nov. (paratype). D. L. maai Wittmer, 1973 (paratype). E. L. marchei Pic, 1922. F. L. politus Fairmaire, 1880. G. L. sabangensis Wittmer, 1985 (paratype). H. L. sericatus Champion, 1924. I. L. submariniformis Wittmer, 1985 (paratype). 
Female

HL/HW 0.79-0.86 (0.81); PW/PL 1.16-1.21 (1.17); EL/EW 1.42-1.47 (1.45); EL/PL 2.61-2.78 (2.70); EW/PW 1.57-1.62 (1.59); TL/EW 2.36-2.47 (2.42). Antennae (Fig. 7G) short; antennomere III short, $\mathrm{L} / \mathrm{W}=2.5$; approximate ratio of each antennomeres $(\mathrm{n}=1)$ as $8.0: 1.0: 5.4: 2.0: 2.2: 2.4: 2.6: 2.2$ : $2.2: 2.2: 3.4$.

\section{Measurements}

Male (n = 4): TL 4.03-4.73 (4.23) mm; HL 0.80-0.98 (0.85) mm; HW 0.90-1.08 (0.97) mm; PW 1.021.20 (1.09) mm; PL 0.88-1.05 (0.94) mm; EL 2.35-2.70 (2.44) mm; EW 1.57-1.90 (1.71) mm. Female $(\mathrm{n}=4)$ : TL 4.18-4.30 (4.23) mm; HL 0.75-0.80 (0.76) mm; HW 0.92-0.95 (0.94) mm; PW 1.05-1.15 (1.10) mm; PL 0.90-0.95 (0.94) mm; EL 2.48-2.60 (2.53) mm; EW 1.70-1.82 (1.75) mm.

\section{Distribution}

Philippines (Palawan).

Laius sericatus Champion, 1924

Figs $3 \mathrm{H}, 6 \mathrm{E}, 7 \mathrm{H}, 8 \mathrm{H}, 9 \mathrm{H}$

Laius sericatus Champion, 1924: 301.

\section{Material examined}

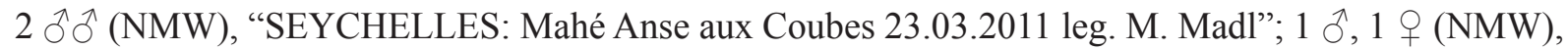
ditto but "21.03.2011"; 3 ㅎํ, 2 우우 (1 우 in ethanol; NMW, EUMJ), "SEYCHELLES: Mahé Petite Police intertidal 28.10.2010, leg. M. Madl”.

\section{Redescription}

Male

HL/HW 0.74-0.75 (0.75); PW/PL 1.27-1.31 (1.29); EL/EW 1.40-1.52 (1.47); EL/PL 2.90-3.04 (2.95); EW/PW 1.54-1.58 (1.56); TL/EW 2.27-2.42 (2.37). Antennae (Fig. 7H) long; antennomere I stout; antennomere III (Fig. 8H) transverse, shallowly and narrowly concave in dorsal portion, L/W = 0.72; approximate ratio of each antennomere $(\mathrm{n}=1)$ as $6.6: 1.0: 4.7: 1.7: 1.7: 1.6: 1.6: 1.6: 1.6: 1.6$ : 2.6. Caudal margin of tergite VII concave. Aedeagus (Fig. 9H) about $1.22 \mathrm{~mm}$, round at aedeagal apex. Gonoporal piece (Fig. 3H) straight, wide, $0.62 \mathrm{~mm}$ in GL; GL/AL 2.11. Ligula (Fig. 3H) short, slightly curved; LL 0.24 mm; LW 0.09 mm; LL/LW 2.69; GL/LL 2.58.

\section{Female}

HL/HW 0.77-0.92 (0.85); PW/PL 1.27-1.29 (1.28); EL/EW 1.41-3.53 (2.47); EL/PL 2.73-2.89 (2.81); EW/PW 0.61-1.59 (1.10); TL/EW 2.37-5.82 (4.10). Antennae (Fig. 7H) long; antennomere III relatively long, $\mathrm{L} / \mathrm{W}=2.73$; approximate ratio of each antennomeres $(\mathrm{n}=1)$ as $8.0: 1.0: 4.8: 2.0: 2.0: 2.0: 2.0$ : $2.0: 2.0: 2.0: 3.4$.

\section{Measurements}

Male (n = 3): TL 5.20-5.55 (5.43) mm; HL 0.90-0.95 (0.92) mm; HW 1.20-1.28 (1.23) mm; PW 1.401.55 (1.47) mm; PL 1.10-1.18 (1.14) mm; EL 3.20-3.50 (3.37) mm; EW 2.15-2.45 (2.30) mm. Female $(\mathrm{n}=2)$ : TL 4.62-4.95 (4.79) mm; HL 0.85-0.92 (0.89) mm; HW 1.00-1.10 (1.05) mm; PW 1.23-1.40 (1.32) mm; PL 0.95-1.10 (1.03) mm; EL 2.75-3.00 (2.88) mm; EW 0.85-1.95 (1.40) mm.

\section{Distribution}

Seychelles (Mahé, Félicité Isls.). 
Laius submariniformis Wittmer, 1985

Figs 3I, 6F, 7I, 8I, 9I

Laius submariniformis Wittmer, 1985: 385.

\section{Material examined}

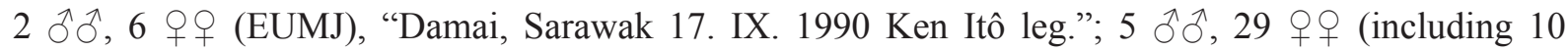
paratypes; EUMJ), "PHILIPPINES Sabang, N. of Mt. St. Paul, Palawan July, 11-13, 1977 M. Sato leg."; 5 ふふર, 4 웅 (BPBM), "PHILIPPINES (S.) Cagayan Sulu I. Feb. 27. 1957”, "Yoshio Kondo collector”.
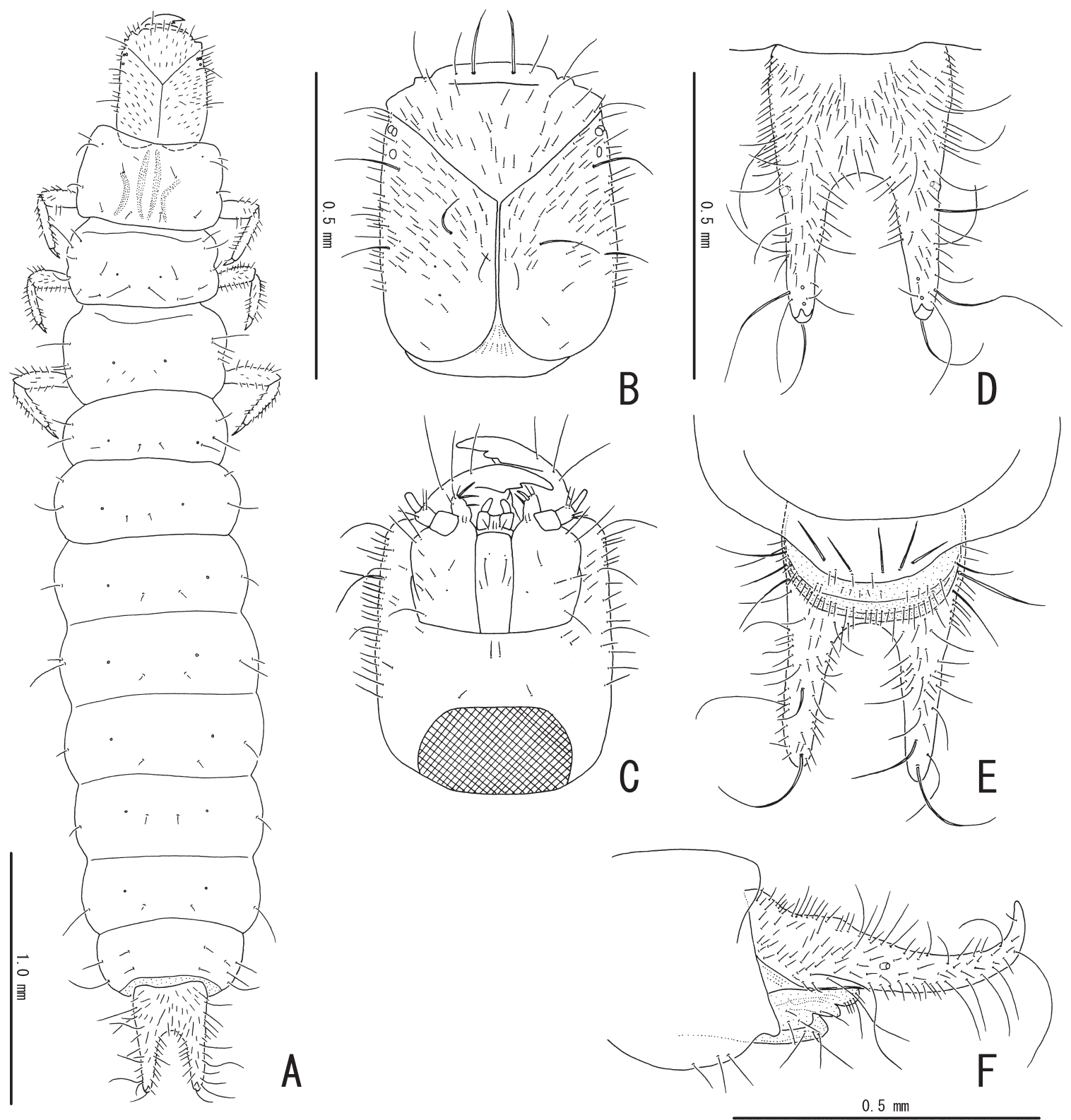

Fig. 10. Larva of Laius rodriguesensis sp. nov. A. Dorsal habitus. B. Head in dorsal view. C. Head in ventral view. D. Abdominal segment IX in dorsal view. E. Abdominal segment IX in ventral view. F. Abdominal segment IX in lateral view. 


\section{Redescription}

Male

HL/HW 0.75-0.86 (0.80); PW/PL 1.27-1.37 (1.32); EL/EW 1.38-1.50 (1.42); EL/PL 2.64-2.94 (2.79); EW/PW 1.43-1.56 (1.48); TL/EW 2.25-2.49 (2.37). Antennae (Fig. 7I) long; antennomere I long and slightly curved laterally; antennomere III (Fig. 8I) oval, deeply concave in dorso-mesal portion, L/W = 0.88 ; approximate ratio of each antennomeres $(\mathrm{n}=1)$ as $15.0: 1.0: 9.0: 3.3: 3.3: 3.0: 3.3: 3.3: 3.3$ : 3.3 : 5.0. Caudal margin of tergite VII deeply concave. Aedeagus (Fig. 9I) about $1.37 \mathrm{~mm}$, concave at aedeagal apex. Gonoporal piece (Fig. 3I) straight, rather wide, with very short basal projection, $0.77 \mathrm{~mm}$ in GL; GL/AL 1.51. Ligula (Fig. 3I) long, evenly curved; LL 0.37 mm; LW 0.14 mm; LL/LW 2.58; GL/ LL 2.06.

\section{Female}

HL/HW 0.67-0.85 (0.76); PW/PL 1.32-1.45 (1.36); EL/EW 1.33-1.52 (1.39); EL/PL 2.64-2.91 (2.75); EW/PW 1.31-1.59 (1.46); TL/EW 2.15-2.48 (2.31). Antennae (Fig. 7I) long; antennomere III short, $\mathrm{L} / \mathrm{W}=2.14$; approximate ratio of each antennomeres $(\mathrm{n}=1)$ as $8.0: 1.0: 4.2: 2.3: 2.0: 2.0: 2.0: 1.8$ : $1.8: 1.8: 3.3$.

\section{Measurements}

Male (n = 4): TL 4.90-6.33 (5.60) mm; HL 0.88-1.20 (1.02) mm; HW 1.05-1.52 (1.29) mm; PW 1.401.82 (1.60) mm; PL 1.02-1.40 (1.21) mm; EL 3.00-3.78 (3.37) mm; EW 2.00-2.70 (2.37) mm. Female (n = 5): TL 4.95-5.65 (5.30) mm; HL 0.80-1.10 (0.95) mm; HW 1.20-1.32 (1.24) mm; PW 1.45-1.68 (1.58) mm; PL 1.10-1.25 (1.16) mm; EL 3.05-3.30 (3.19) mm; EW 2.10-2.40 (2.30) mm.

\section{Distribution}

Philippines (Palawan, Sulu Isls.), Malaysia (Sarawak).

\section{Remarks}

This is the first record of this species from Sulu Island and Borneo.

Laius asahinai Nakane, 1955

Figs 3J, 13D

\section{Material examined}

JAPAN: 1 q (EUMJ), “Oshima, Okada, Shinkai, Nodahama, 31. V. 2013, H. Kamezawa leg.”; 1 q (EUMJ), “[IZ60] Ookubohama, Miyakejima, Izu Isls., N34 07.272 E139 30.930, ca 152 m, 29. VI. 2013, H. Yoshitomi leg.”; 1 §̂, 9 우 (EUMJ), “[IZ58] Sabigahama, Miyake-jima, Izu Isls., N34 04.241 E139 28.837, ca 151 m, 28-30. VI. 2013, H. Yoshitomi leg."; 1 q (EUMJ), "Shimo-Koshiki Is. Kagoshima,

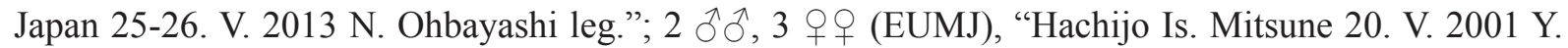
Kawabata". Others see Yoshitomi \& Lee (2010).

\section{Description of endophallic sclerites}

Gonoporal piece (Fig. 3J) straight, slender, $0.81 \mathrm{~mm}$ in GL; GL/AL 3.96. Ligula (Fig. 3J) short, slightly curved; LL 0.17 mm; LW 0.04 mm; LL/LW 3.88; GL/LL 4.71.

\section{Distribution}

Japan (Honshu, Izu Isls. [Oshima, Miyake-jima, Hachijo-jima], Shikoku, Kyushu, Shimo-koshiki-jima, Yakushima). 

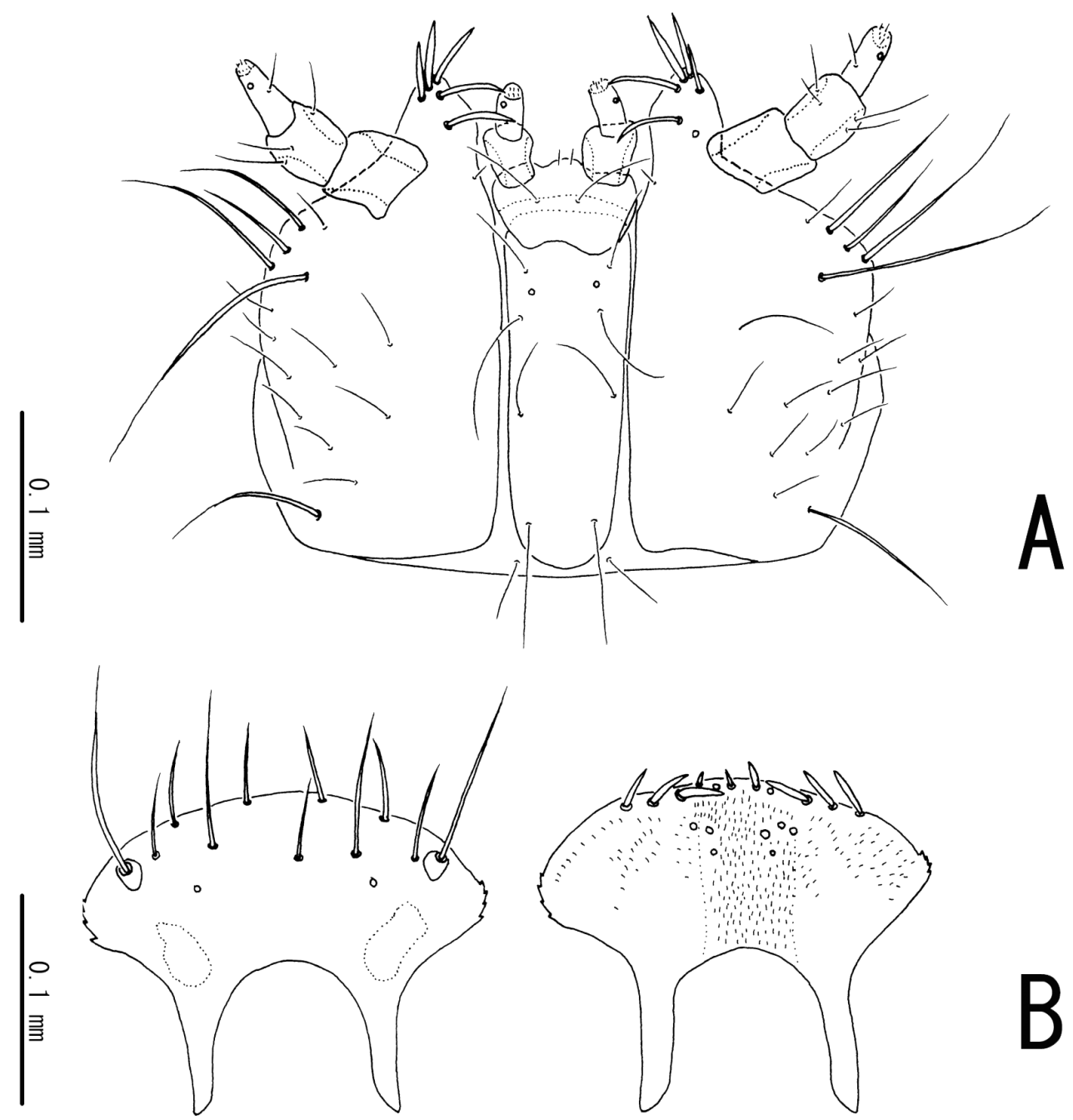

A
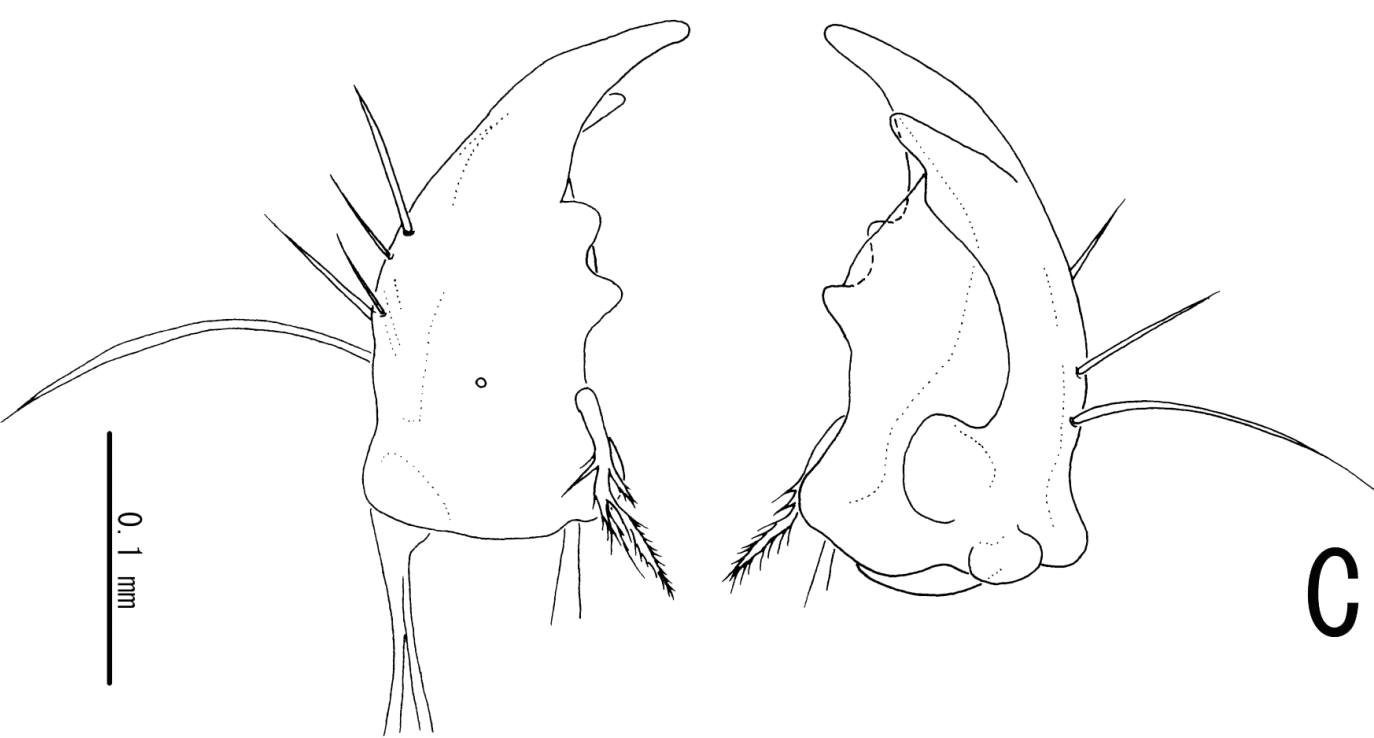

Fig. 11. Larva of Laius rodriguesensis sp. nov. A. Maxillae and labium in ventral view. B. Labrum in dorsal (left) and ventral (right) views. C. Mandible in dorsal (left) and ventral (right) views. 


\section{Remarks}

This is the first record of this species from Oshima, Miyake-jima, and Shimo-koshiki-jima. This species is distributed in the northernmost area in the genus, and widely spread. The local variation of the endophallic sclerites was not observed in this study, but the specimens in the Izu Isls. have somewhat darker elytral coloration.

Laius etsukoae Satô, Yoshitomi \& Ohbayashi, 2006

Fig. 4A

\section{Material examined}

Same as in Satô et al. (2006).

\section{Description of endophallic sclerites}

Gonoporal piece (Fig. 4A) straight, slender, $0.59 \mathrm{~mm}$ in GL; GL/AL 2.49. Ligula (Fig. 4A) short, distinctly curved in apical portion; LL $0.24 \mathrm{~mm}$; LW $0.10 \mathrm{~mm}$; LL/LW 2.39; GL/LL 2.49. Additional sclerite (Fig. 4A) present, $0.05 \mathrm{~mm}$.

\section{Laius flavicornis (Fabricius, 1801)}

Fig. 4B

\section{Material examined}

Same as in Yoshitomi (2008).

\section{Description of endophallic sclerites}

Gonoporal piece (Fig. 4B) straight, slender, with short and curved basal projection, $0.74 \mathrm{~mm}$ in GL; GL/ AL 2.14. Ligula (Fig. 4B) short, strongly curved in half; LL $0.26 \mathrm{~mm}$; LW $0.12 \mathrm{~mm}$; LL/LW 2.14; GL/ LL 2.85 .

Laius keiichii Satô, Yoshitomi \& Ohbayashi, 2006

Fig. 4C

\section{Material examined}

Same as in Satô et al. (2006).

\section{Description of endophallic sclerites}

Gonoporal piece (Fig. 4C) straight, slender, $0.54 \mathrm{~mm}$ in GL; GL/AL 1.48. Ligula (Fig. 4C) long and slender, evenly and strongly curved; LL $0.31 \mathrm{~mm}$; LW $0.16 \mathrm{~mm}$; LL/LW 1.90; GL/LL 1.76. Additional sclerite (Fig. 4C) present. $0.04 \mathrm{~mm}$.

Laius lutaoensis Yoshitomi \& Lee, 2010

Figs 2, 4D

\section{Material examined}

$3 \widehat{\jmath} \widehat{\jmath}, 7$ q $q$ (EUMJ), “Taitung, TAIWAN Is. Ludao 4-6. IV. 2012 N. OHBAYASHI leg.”. Other material studied is already mentioned in Yoshitomi \& Lee (2010).

\section{Description of endophallic sclerites}

Gonoporal piece (Fig. 4D) straight, slender, $0.64 \mathrm{~mm}$ in GL; GL/AL 2.09. Ligula (Fig. 4D) short, strongly curved in half; LL $0.23 \mathrm{~mm}$; LW $0.12 \mathrm{~mm}$; LL/LW 2.00; GL/LL 2.74. 
Laius madli Yoshitomi, 2010

Fig. 4E

\section{Material examined}

Same as in Yoshitomi (2010).

\section{Description of endophallic sclerites}

Gonoporal piece (Fig. 4E) curved, rather wide, $0.63 \mathrm{~mm}$ in GL; GL/AL 2.10. Ligula (Fig. 4E) relatively short, curved in basal portion; LL 0.28 mm; LW 0.09 mm; LL/LW 2.94; GL/LL 2.26.

\section{Laius miyamotoi Nakane, 1955}

Fig. $4 \mathrm{~F}$

\section{Material examined}

Same as in Yoshitomi \& Lee (2010).

\section{Description of endophallic sclerites}

Gonoporal piece (Fig. 4F) straight, slender, $0.72 \mathrm{~mm}$ in GL; GL/AL 4.13. Ligula (Fig. 4F) short, strongly curved in half; LL 0.15 mm; LW 0.08 mm; LL/LW 1.80; GL/LL 4.81.
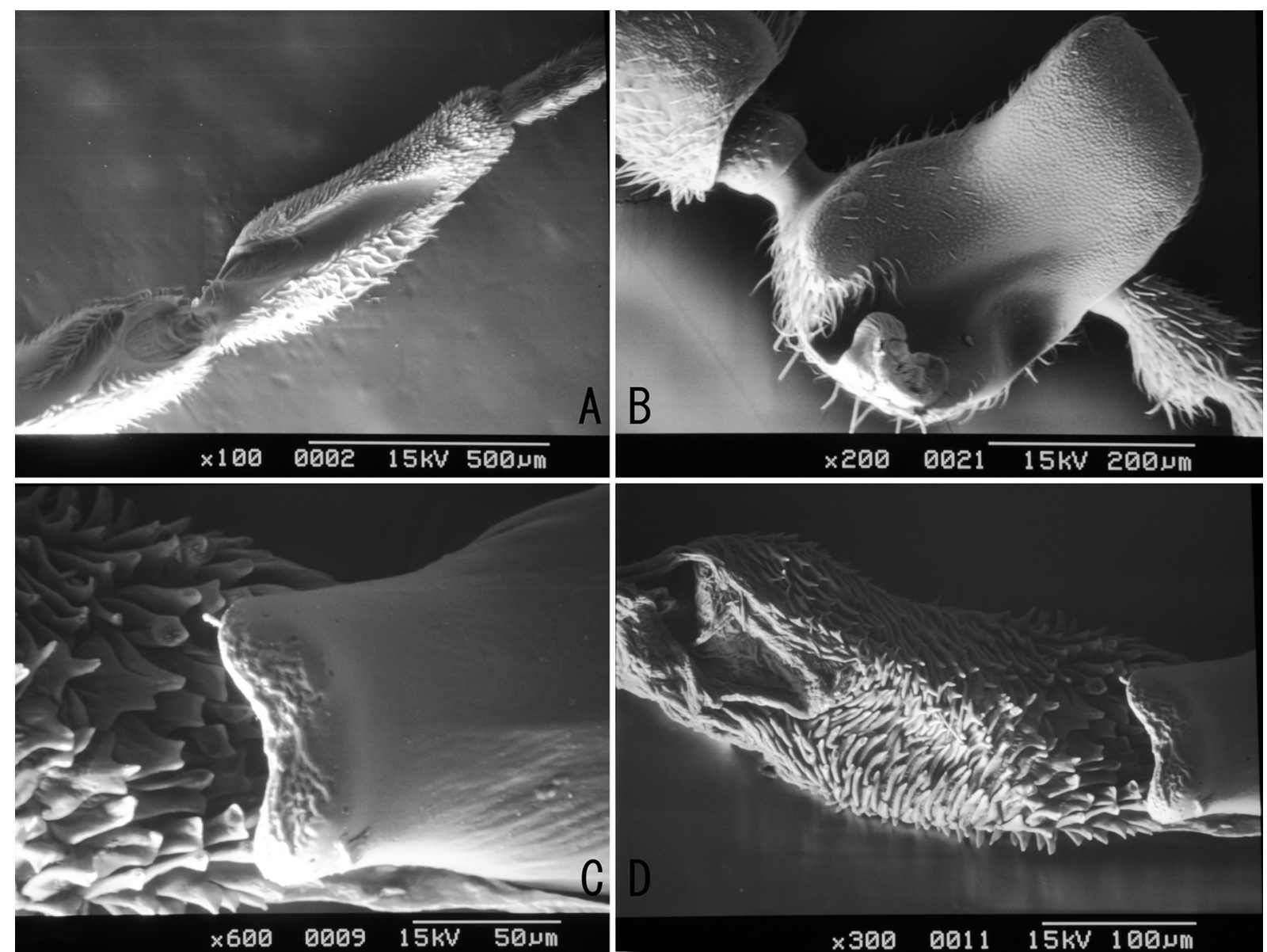

Fig. 12. Laius andamanensis sp. nov., $\widehat{\jmath}$, paratype. A. Inner part of fore leg, showing enlarged femur and tibia. B. Antennomere III. C. Aedeagal apex. D. Spinous area (SEM photographs). 
Laius pankowi Wittmer, 1999

Fig. 4I

\section{Material examined}

Same as in Yoshitomi (2008).

\section{Description of endophallic sclerites}

Gonoporal piece (Fig. 4I) curved, wide, $1.04 \mathrm{~mm}$ in GL; GL/AL 0.85. Ligula (Fig. 4I) long and straight, with reticulate and rounded apex; LL 0.77 mm; LW 0.09 mm; LL/LW 8.18; GL/LL 1.35.

\section{Laius satoi Yoshitomi, 2008}

Figs 4G, 13C

\section{Material examined}

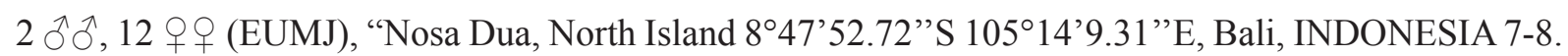
VIII. 2010 H. Yoshitomi leg."; 1 क (EUMJ), "Nosa Dua, South Island 848’6.81”S 115¹4'16.07”E, Bali, INDONESIA 8. VIII. 2010 H. Yoshitomi leg.". Other material studied is already mentioned in Yoshitomi (2008).
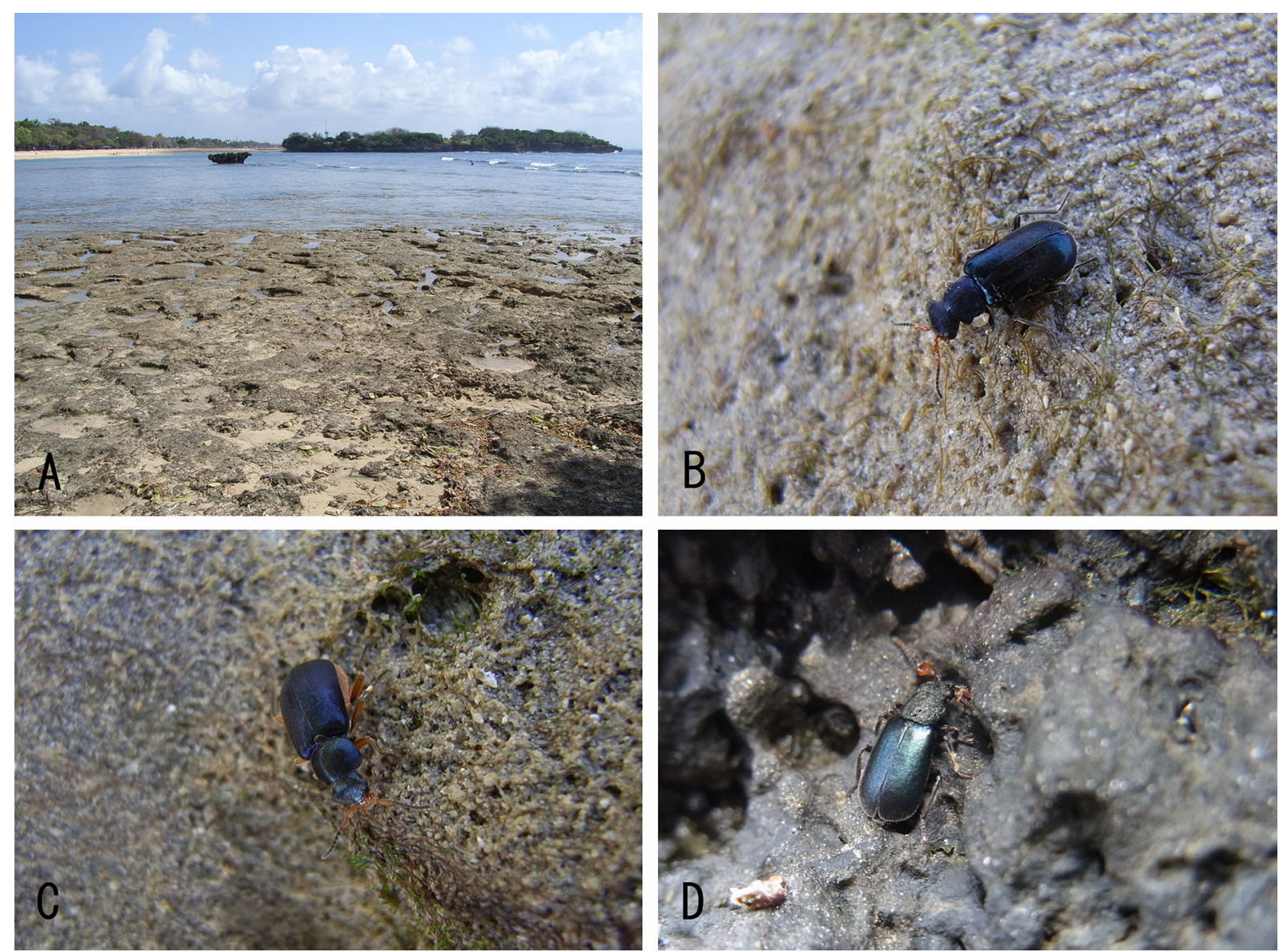

Fig. 13. Habitat and habits of Laius spp. A. Type locality (Nusa Dua, Bali, Indonesia) of Laius baliensis sp. nov. and L. satoi Yoshitomi, 2008, three Laius species living sympatrically. B. L. baliensis sp. nov., female. C. L. satoi, male. D. L. asahinai Nakane, 1955 (at Miyake-jima). Photographs by HY. 


\section{Description of endophallic sclerites}

Gonoporal piece (Fig. 4G) straight, slender, $0.94 \mathrm{~mm}$ in GL; GL/AL 1.25. Ligula (Fig. 4G) long, slightly curved apical portion; LL 0.49 mm; LW 0.07 mm; LL/LW 7.33; GL/LL 1.92.

Laius taiwanus Yoshitomi \& Lee, 2010

Fig. $4 \mathrm{H}$

\section{Material examined}

1 ○े (EUMJ), “Taitung, TAIWAN Is. Ludao 4-6. IV. 2012 N. OHBAYASHI leg.”. Other material studied is already mentioned in Yoshitomi \& Lee (2010).

\section{Description of endophallic sclerites}

Gonoporal piece (Fig. 4H) curved, rather wide, $0.67 \mathrm{~mm}$ in GL; GL/AL 1.68. Ligula (Fig. 4H) long, slightly curved; LL 0.38 mm; LW 0.08 mm; LL/LW 4.60; GL/LL 1.74. Additional sclerite (Fig. 4H) present, $0.09 \mathrm{~mm}$.

\section{Remarks}

Yoshitomi \& Lee (2010) pointed out that this species has local variations between the populations from mainland Taiwan, Lutao and Lanyu Isls. in body size and elytral luster. For the present study, I examined the endophallic structures of these populations, and it became clear that they certainly belong to the same species because of the highly similar features of the endophallic structures.

\section{Discussion}

\section{The endophallic structure of the genus Laius}

The results in this paper show that the endophallic structures of the genus Laius are important taxonomically. For close and precise examination of the endophallic sclerites, it is necessary to remove the sclerites from the aedeagus directly or extract them from the ventral part of the aedeagal apex.

Based on the constructions of endophallic sclerites in 19 species, the genus is subdivided into the following five species groups:

Group 1 (sericatus, etsukoae, keiichii, taiwanus, sabangensis): additional sclerite present.

Group 2 (maai, marchei, submariniformis, flavicornis): gonoporal piece with basal projection.

Group 3 (baliensis sp. nov., andamanensis sp. nov., rodriguesensis sp.nov., politus, sericatus, asahinai, lutaoensis, miyamotoi, madli): ligula short, curved.

Group 4 (satoi): ligula long and straight; gonoporal piece long.

Group 5 (pankowi): ligula long, straight, microcuticulate in apical portion.

\section{Distribution}

The species of Laius inhabit rocky seashores (Fig. 13A), and 1-3 species are distributed in the same place. Table 1 shows the distribution pattern in five areas (Japan, Taiwan, Palawan Isl., Palau, and Bali Isl.). Two species in Taiwan (Lutao Isl.) and the Philippines (Palawan Isl.), and three in Indonesia (Bali Isl.) are sympatric, and have different body sizes and endophallic structures (= different species group) in each area. On the other hand, two species in Japan and Palau are allopatric (or parapatric), their body sizes overlap and the endophallic structures are also similar to each other (= same species group) in each area.

All members of Laius have developed hind wings and are capable of flying, and in fact they often fly in the field. In addition, they are able to stay submerged in seawater for up to 30 hours (Hashimoto 
Table 1. Distribution of Laius spp. in five areas.

\begin{tabular}{|c|c|c|c|c|c|c|c|c|c|}
\hline \multicolumn{2}{|c|}{ Distribution } & \multirow{2}{*}{ Pattern } & \multirow{2}{*}{ Species } & \multirow{2}{*}{$\begin{array}{l}\text { Species } \\
\text { group }\end{array}$} & \multicolumn{4}{|c|}{ Measurement in male $(\mathrm{mm})$} & \multirow{2}{*}{ Reference } \\
\hline & Locality & & & & TL & $\mathbf{A L}$ & GL & $\mathbf{L L}$ & \\
\hline \multirow{2}{*}{ Japan } & $\begin{array}{l}\text { Honshu to } \\
\text { Yakushima }\end{array}$ & allopatric & asahinai & 3 & $3.95-4.65(4.27)$ & 1.19 & 0.81 & 0.17 & $\begin{array}{c}\text { Yoshitomi \& Lee } \\
2010\end{array}$ \\
\hline & Ryukyu Isls. & & miyamotoi & 3 & $3.50-4.20(3.88)$ & 1.17 & 0.72 & 0.15 & $\begin{array}{c}\text { Yoshitomi \& Lee } \\
2010 \\
\end{array}$ \\
\hline \multirow{2}{*}{ Taiwan } & Lutao Isl. & sympatric & taiwanus & 1 & $3.90-4.88(4.18)$ & 1.03 & 0.67 & 0.38 & $\begin{array}{c}\text { Yoshitomi \& Lee } \\
2010\end{array}$ \\
\hline & & & lutaoensis & 3 & $4.80-5.45(5.18)$ & 1.31 & 0.64 & 0.23 & $\begin{array}{c}\text { Yoshitomi \& Lee } \\
2010 \\
\end{array}$ \\
\hline \multirow{2}{*}{ Philippines } & Palawan & sympatric & submariniformis & 2 & $4.90-6.33(5.60)$ & 1.37 & 0.77 & 0.37 & present study \\
\hline & & & sabangensis & 1 & $4.03-4.73(4.23)$ & 0.94 & 0.72 & 0.41 & present study \\
\hline \multirow{2}{*}{ Palau } & Merir, Sonsorol Isls. & allopatric & etsukoae & 1 & $3.4-4.5$ & 1.00 & 0.59 & 0.24 & Satô et al. 2006 \\
\hline & Peleliu Isl. & & keiichii & 1 & 3.8 & 1.19 & 0.54 & 0.31 & Satô et al. 2006 \\
\hline \multirow{3}{*}{ Indonesia } & Bali Isl. & sympatric & satoi & 4 & 5.7 & 1.53 & 0.94 & 0.49 & Yoshitomi 2008 \\
\hline & & & pankowi & 5 & $6.13-6.55(6.34)$ & 1.58 & 1.04 & 0.77 & Yoshitomi 2008 \\
\hline & & & baliensis & 3 & $4.10-4.46(4.27)$ & 1.12 & 0.61 & 0.23 & present study \\
\hline
\end{tabular}

1970). Therefore it is supposed that the distribution of each species is widely spread through flight and tidal currents. However, most of the species show narrow distributions (see Appendix). The genus Aegialites Mannerheim, 1853 (Coleoptera, Salpingidae, Aegialitinae) also lives in rocky seashores, and the distribution of each of its species is generally restricted as the ones of the species of the genus Laius (see Doyen 1976; Zerche 2004). However, Aegialites is flightless with degenerated hind wings, and its distribution is limited in the Northern Pacific (latitude c. $30-60^{\circ} \mathrm{N}$; Northern Japan, Kuril Isls., to North America). The speciation and distribution pattern of Laius provide interesting zoogeographical and evolutionary themes.

\section{Acknowledgements}

I thank Dr. Manfred Jäch (NMW), Dr. Michael Geiser (NHMB), Mr. Ryo Ogawa (Kobe Univ.), Dr. Junsuke Yamasako and Dr. Nobuo Ohbayashi (both EUMJ), Miss Makoto Asano, Mr. Shepherd Myers (BPBM), Dr. Nugroho Susetya Putra (Gadjah Mada University), and the late Dr. Masataka Satô for supplying the materials used in this paper, and Mr. Dennis Murphy (The United Graduate School of Agricultural Sciences, Ehime University) for his critical reading of the draft. A part of this study is supported by the KAKENHI (24510333; head investigator: H. Kojima).

\section{References}

Asano M. \& Kojima H. 2009. On the Mature Larvae of the Genus Laius (Coleoptera, Malachiidae) from Japan. Japanese Journal of Systematic Entomology 15 (2): 481-486.

Champion G.C. 1921. XXXVII.--Notes on various African and Asiatic Species of Laius Guérin, with an Account of their accessory -characters [Coleoptera]. The Annals and Magazine of Natural History serie 9 7: 322-343. http://dx.doi.org/10.1080/00222932108632526

Champion G.C. 1924. Coleoptera from the Seychelles: Lampyridae, Helodidae, Cantharidae, Melyridae, and supplement to Cleridae. Transactions of the Royal Entomological Society of London 1923 (3-4): 295-304. 
Doyen J.T. 1976. Chapter 18. Marine beetles (Coleoptera excluding Staphylinidae). In: Cheng L. (ed.) Marine insects: 497-519. North-Holland Publishing Company, Amsterdam.

Evers A.M.J. 1994. Zur Phylogenie von Laius Guér., Collops Er. und der verwandten Gattungen. Entomologische Blätter für Biologie und Systematik der Käfer 90: 169-181.

Fairmaire L. 1880. Diagnoses de coléoptères de Nossi-Bé. Le Naturaliste 2: 246.

Guérin-Méneville F.E. 1830. Crustacés, Arachnides et Insectes. In: Duperrey M.L.I. (ed.) Voyage autour du monde: exécuté par ordre du roi, sur la corvette de Sa Majesté, la Coquille, pendant les années 1822, 1823, 1824, et 1825. Tome II. Part. 2 (1): 1-319. Arthus Bertrand, Paris. http://dx.doi.org/10.5962/bhl. title. 57936

Hashimoto H. 1970. Insects in the sea. Insectarium 7: 15. (in Japanese, with English title)

Kôno H. \& Esaki T. 1937. Ein Neuer, halophiler Käfer, Rotamalachius (n.g.) esakii (n.sp.) Kôno (Coleoptera, Malachiidae). Annotationes Zoologicae Japonenses 16 (2): 121-123.

Nakane T. 1955. Marine insects of the Tokara Islands. V. Three new species of the genus Laius Guérin from Kyushu and the Tokara Islands, with notes on a species from Marianna. Publications of the Seto Marine Biological Laboratory 4 (2-3): 373-378.

Pic M. 1922. Coléoptères Malacodermes nouveaux des Collections du Muséum. Bulletin du Muséum national d'Histoire naturelle (Paris) 28 (2): 157-161.

Plonski I.S. 2013. Studies on the genus Intybia Pascoe (Coleoptera: Malachiidae). I. Some nomenclatorial acts and faunistic records. Zeitschrift der Arbeitsgemeinschaft Österreichischer Entomologen 65: 61-68.

Sasakawa K. 2005. Phylogenetic Studies of the Subgenus Nialoe (s. lat.) (Coleoptera, Carabidae, Genus Pterostichus), Part 1: Homology of the Component Parts of Male Genitalia and Higher Phylogeny. Zoological Science 22: 1205-1216. http://dx.doi.org/10.2108/zsj.22.1205

Satô M. 1964. Studies on the marine beetles in Japan, IV: Some remarks on the fauna of marine beetles in Japan. Journal of the Nagoya Jogakuin College 10: 76-81.

Satô M., Yoshitomi H. \& Ohbayashi T. 2006. Notes on Melyridae (Coleoptera) of Micronesia. Elytra 34: 343-351.

Sharp D. \& Muir F. 1912. The comparative anatomy of the male genital tube in Coleoptera. Transactions of the Entomological Society of London 1912: 477-642. http://dx.doi.org/10.5962/bhl.title.11474

Simmons L.W. 2014. Sexual selection and genital evolution. Austral Entomology 53 (1): 1-17. http:// dx.doi.org/10.1111/aen.12053

Westwood J.O. 1833. XXXIV. On the Paussidae, a family of coleopterous insects. Transactions of the Linnean Society of London 16 (3): 607-684. http://dx.doi.org/10.1111/j.1095-8339.1829.tb00157.x

Wittmer W. 1958. Coleoptera: Lampyridae, Cantharidae, Malachiidae, and Prionoceridae. Insects of Micronesia 16 (2): 67-74.

Wittmer W. 1973. Zur Kenntnis der Malachiidae (Col.) von Neuguinea, Neubritannien, Newireland und den Salomonen. Pacific Insects 15 (3-4): 289-352.

Wittmer W. 1985. 38. Beitrag zur Kenntnis der indo-malaiischen Fauna. Entomologische Arbeiten aus dem Museum G. Frey 33-34: 381-390.

Wittmer W. 1997. Zur Kenntnis der Gattungen Intybia Pascoe und Stenolaius Wittmer (Coleoptera, Malachiidae). Japanese Journal of Systematic Entomology 3 (2): 181-211. 
Yamasako J. \& Ohbayashi N. 2011. Review of the genus Paragolsinda Breuning, 1956 (Coleoptera, Cerambycidae, Lamiinae, Mesosini), with reconsideration of the endophallic terminology. Zootaxa 2882: 35-50.

Yoshitomi H. 2008. Contribution to the taxonomy of the genus Laius Guérin-Menevelle in Indonesia, with description of a new species (Coleoptera: Malachiidae). Koleopterologische Rundschau 78: 285290.

Yoshitomi H. 2010. A new species of the genus Laius (Coleoptera, Malachiidae) from Mauritius. Japanese Journal of Systematic Entomology 16 (1): 1-4.

Yoshitomi H. \& Lee C.-F. 2010. Revision of the Taiwanese and Japanese species of the genus Laius (Insecta: Coleoptera: Malachiidae). Zoological studies 49 (4): 534-543.

Zerche L. 2004. Revision der Gattung Aegialites Mannerheim (Coleoptera: Salpingidae: Aegialitinae). Stuttgarter Beiträge zur Naturkunde Series A (Biologie) 666: 1-116.

Manuscript received: 22 March 2014

Manuscript accepted: 25 June 2014

Published on: 25 September 2014

Topic editor: Koen Martens

Desk editor: Charlotte Thionois

Printed versions of all papers are also deposited in the libraries of the institutes that are members of the EJT consortium: Muséum National d'Histoire Naturelle, Paris, France; Botanic Garden Meise, Belgium; Royal Museum for Central Africa, Tervuren, Belgium; Natural History Museum, London, United Kingdom; Royal Belgian Institute of Natural Sciences, Brussels, Belgium; Natural History Museum of Denmark, Copenhagen, Denmark. 
Appendix. Updated list of the true Laius species of the world. * Laius heterocerus Boisduval, 1835 was treated as a junior synonym of this species, and recorded from New Guinea, India, Key Isls., Karoline, New Caledonia (Evers 1994). ** This species has also been recorded from Larat, Key Isls., Borneo, Lundu, Sarawak, and China (Evers 1994); however, these records were probably based on the misidentification of another species.

Laius alleni Lea, 1909 Australia (Queensland) [after Evers 1994]

Laius ambonensis Wittmer, 1996 Indonesia (Ambon Isl.) [after Plonski 2013]

Laius andamanensis Yoshitomi sp. nov. India (Andaman Islands)

Laius asahinai Nakane, 1955 Japan (Honshu to Yakushima)

Laius baliensis Yoshitomi sp. nov. Indonesia (Bali Isl.)

Laius cyaneus Guérin-Méneville, 1830* .India, New Guinea [after Evers 1994])

Laius cyanoxanthus Bourgeois, 1897 .New Hebrides (Mallicolo) [after Evers 1994]

Laius etsukoae Satô, Yoshitomi \& Ohbayashi, 2006

Laius flavicornis (Fabricius, 1801)** Palau (Merir, Sonsorol Isls.)

Laius impressus Wittmer, 1985 Indonesia (Java Isl.)

Laius keiichii Satô, Yoshitomi \& Ohbayashi, 2006 Philippines (Mindanao Isl.) [after Evers 1994]

Laius lutaoensis Yoshitomi \& Lee, 2010. .Palau (Peleliu Isl.)

Laius miyamotoi Nakane, 1955. Taiwan

Laius maai Wittmer, 1973 Japan (Ryukyu Isls.)

Laius madli Yoshitomi, 2010 New Guinea

Laius marchei Pic, 1922 Republic of Mauritius (Mauritius Isl.)

Laius miles Bourgeois, 1905 Mariana (Rota, Guam Isls.)

Laius mindanaonus Wittmer, 1985 Ceylon [after Evers 1994]

Laius obscurus (Pic, 1908) Philippines (Mindanao Isl.)

Laius pankowi Wittmer, 1999 Kenya [after Evers 1994]

Laius politus Fairmaire, 1880 Indonesia (Bali Isl.)

Laius purpureipennis Lea, 1916 Madagascar

Laius riedeli Evers, 1994 Australia [after Evers 1994]

Laius rodriguesensis Yoshitomi sp. nov. Malaysia (Sarawak)

Laius rufipes Montrouzier, 1860. Republic of Mauritius (Rodrigues Isl.)

Laius sabangensis Wittmer, 1985 .New Caledonia [after Evers 1994]

Laius satoi Yoshitomi, 2008 Philippines (Palawan Isl.)

Laius sericatus Champion, 1923. Indonesia (Bali Isl.)

Laius submarinus Champion, 1921 Seychelles (Mahé, Félicité Isls.)

Laius submariniformis Wittmer, 1985 Philippines (Mindanao Isl.), New Guinea

Laius taiwanus Yoshitomi \& Lee, 2010 Philippines (Palawan Isl.)

Laius tibialis Gahan, 1900 Taiwan (including Lutao Isls.)

Laius velutinus Gerstaecker, 1873 Christmas Isl.

Laius wallecei Pic, 1937 Zanzibar Coast [after Champion 1921] Malaysia (Borneo) 